\title{
Stability analysis of switched homogeneous time-delay systems under synchronous and asynchronous commutation*
}

\author{
A. Aleksandrov ${ }^{\dagger}$, D. Efimov ${ }^{\ddagger \S}$
}

August 4, 2021

\begin{abstract}
In this work, stability analysis for a class of switched nonlinear time-delay systems is performed by applying Lyapunov-Krasovskii and Lyapunov-Razumikhin approaches. It is assumed that each subsystem in the family is homogeneous (of positive or negative degree) and asymptotically stable in the delay-free setting. The cases of existence of a common or multiple Lyapunov-Krasovskii functionals and a common Lyapunov-Razumikhin function are explored. The scenarios with synchronous and asynchronous switching are considered, and it is demonstrated that depending on the kind of commutation, one of the frameworks for stability analysis outperforms another, but finally leading to similar restrictions for both types of switching (despite the asynchronous one seems to be more demanded). The obtained results are applied to mechanical systems having restoring forces with real-valued powers.
\end{abstract}

Keywords: Switched systems, Time-delay systems, Homogeneous systems, LyapunovKrasovskii approach, Lyapunov-Razumikhin approach

\section{Introduction}

Intensive development of large-scale systems, networked multi-agent processes and cyber-physical dynamics puts focus on the role of delays and their influence on performances (stability, convergence rate, robustness) of these systems [29, 37, 28, 47, 31, 11. There are two main approaches for stability analysis of time-delay systems, based on either a Lyapunov-Krasovskii functional or a Lyapunov-Razumikhin function, and these methods are well-investigated for linear dynamics [26], where a wide choice of Lyapunov-Krasovskii functionals and Lyapunov-Razumikhin functions is available, depending on the properties of the delay and the system uncertainty. For some classes of nonlinear plants, as port-Hamiltonian systems [50], for instance, also there exist some results, but in general the nonlinear setting is less studied, and there is no canonical selection of respective functional or function. The common conclusion is that the LyapunovKrasovskii approach is less conservative (it has been also proven to be necessary and sufficient

* This work was partially supported by the Ministry of Science and Higher Education of Russian Federation, passport of goszadanie no. 2019-0898, and by the Russian Foundation for Basic Research (grant no. 19-0100146-a).

†Saint Petersburg State University, 7-9 Universitetskaya nab., 199034 Saint Petersburg, Russia.

‡Univ. Lille, Inria, CNRS, UMR 9189 - CRIStAL, F-59000 Lille, France.

§ITMO University, 49 av. Kronverkskiy, 197101 Saint Petersburg, Russia. 
for stability in many cases) [35, 34], while the Lyapunov-Razumikhin method is more simple to apply in the nonlinear scenario since a delay-free Lyapunov function can be tested.

A promising canonical class of dynamical models is composed by homogeneous systems taking an intermediate place between linear and nonlinear ones [11]. The theory of homogeneous dynamical systems has been developed, first, for ordinary differential equations [60, 36, 10, 13], and next extended to differential inclusions [25, 39, 11], time-delay systems [22, 23, partial differential equations [46] and discrete-time systems [49]. The main features of a homogeneous system are that its local behavior is inherited globally, the homogeneous stable/unstable systems admit homogeneous Lyapunov/Chetaev functions [60, 33, 48, 19, 21, 24] and possess robustness properties with respect to external inputs [12]. Using the concept of local homogeneity [60, 8, 20] a generic dynamical system may be locally approximated by a homogeneous one, which allows the theory of homogeneity to be applied in a larger area. It has been shown that for any value of delay, the homogeneous systems, which are asymptotically stable in the delay-free case, are locally asymptotic stable for positive degree and globally asymptotic stable with respect to a compact set containing the origin for negative degree [16, 9, 45, 15, 5, 6, 23, 59. These results have been obtained using the Lyapunov-Razumikhin [23, 59] and Lyapunov-Krasovskii [7, 17] theories. Moreover, in [17] a canonical form of Lyapunov-Krasovskii functional has been proposed that can be used for any (locally) homogeneous systems. This achievement allows the Lyapunov-Krasovskii approach to be effectively applied for this class of models.

Another interesting for investigation problem, whose solution is demanded in many applications, deals with analysis and design of switched systems [40], which form a subclass of hybrid dynamics. The stability conditions in this setting depend on the kind of commutation: an arbitrary switching, when usually the common Lyapunov function approach is used [41], or (average) dwell-time switching, when a restriction on minimal time between commutation is imposed [30, 51, 52]. For time-delay systems there exist the corresponding extensions in terms of Lyapunov-Krasovskii and Lyapunov-Razumikhin approaches [57, 54, 53, 32, 44, 56.

In the present work, a switched time-delay system is studied, where all subsystems are homogeneous, which have negative or positive homogeneity degree and asymptotic stability property in the delay-free scenario. Two cases are considered with synchronous and asynchronous switching. In the latter scenario there are terms dependent on a delayed commutation signal, then usually more restrictive conditions have to be imposed on the properties of subsystems and commutation signal to substantiate stability. Selecting an arbitrary switching and the common Lyapunov-Krasovskii functional or Lyapunov-Razumikhin function approaches, a comparison of these methods is performed on the chosen class of problems. The multiple LyapunovKrasovskii functionals under commutation time restrictions are also investigated. The obtained theory is applied for stability analysis in a class of mechanical systems with real-valued power restoring forces, where switching is served for representation of their non-smooth behavior. Our comparison of Lyapunov-Krasovskii and Lyapunov-Razumikhin approaches shows that both stability methods have different applicability conditions, outperforming one another depending on a scenario, but finally giving the same stability restrictions for synchronous and asynchronous cases, which is a remarkable finding (even for the linear case [55]). Other novel aspects come from consideration of homogeneous systems of both, positive and negative, degrees, with new Lyapunov-Krasovskii functionals and different kinds of commutation, and application of these results to a class of mechanical systems.

The outline of the paper is as follows. Preliminary results are given in Section 2. The considered stability problem is formulated in Section 3. The common Lyapunov-Razumikhin function or Lyapunov-Krasovskii functional methods are considered in sections 4 and 5, respectively. The multiple Lyapunov-Krasovskii functionals are analyzed in Section 6. Application of the obtained results to a mechanical system is presented in Section 7. An illustration of the 
proposed conditions in simulation example is described in Section 8. The concluding remarks and discussion are summarized in Section 9 ,

\section{Preliminaries}

The real and nonnegative real numbers are denoted by $\mathbb{R}$ and $\mathbb{R}_{+}$, respectively. Denote by $C\left([-\tau, 0], \mathbb{R}^{n}\right)$ the Banach space of continuous functions $\phi:[-\tau, 0] \rightarrow \mathbb{R}^{n}$ with the uniform norm $\|\phi\|_{\tau}=\sup _{-\tau \leq \varsigma \leq 0}\|\phi(\varsigma)\|$, where $\|\cdot\|$ is the standard Euclidean norm. Other used notation is either standard [26] or explained after the first introduction.

The definitions of utilized stability properties can be found in the basic textbooks [29, 37, [26, 35.

A continuous function $\alpha: \mathbb{R}_{+} \rightarrow \mathbb{R}_{+}$belongs to class $\mathcal{K}$ if it is strictly increasing and $\alpha(0)=0$; it belongs to class $\mathcal{K}_{\infty}$ if it is also unbounded.

\subsection{Homogeneity}

For any $r_{i}>0, i=1, \ldots, n$ and $\lambda>0$, define the dilation matrix $\Lambda_{r}(\lambda)=\operatorname{diag}\left\{\lambda^{r_{i}}\right\}_{i=1}^{n}$ and the vector of weights $\mathbf{r}=\left(r_{1}, \ldots, r_{n}\right), r_{\max }=\max _{1 \leq j \leq n} r_{j}$ and $r_{\min }=\min _{1 \leq j \leq n} r_{j}$.

Definition 1. [22] The function $g: C\left([-\tau, 0], \mathbb{R}^{n}\right) \rightarrow \mathbb{R}$ is called $\mathbf{r}$-homogeneous if for any $\phi \in C\left([-\tau, 0], \mathbb{R}^{n}\right)$ the relation

$$
g\left(\Lambda_{r}(\lambda) \phi\right)=\lambda^{d} g(\phi)
$$

holds for some $d \in \mathbb{R}$ and all $\lambda>0$.

The function $f: C\left([-\tau, 0], \mathbb{R}^{n}\right) \rightarrow \mathbb{R}^{n}$ is called $\mathbf{r}$-homogeneous if for any $\phi \in C\left([-\tau, 0], \mathbb{R}^{n}\right)$ the relation

$$
f\left(\Lambda_{r}(\lambda) \phi\right)=\lambda^{d} \Lambda_{r}(\lambda) f(\phi)
$$

holds for some $d \geq-r_{\min }$ and all $\lambda>0$.

In both cases, the constant $d$ is called the degree of homogeneity.

The introduced notion of weighted homogeneity in $C\left([-\tau, 0], \mathbb{R}^{n}\right)$ is reduced to the standard one in $\mathbb{R}^{n}$ if $\tau \rightarrow 0$.

For any $r_{i}>0, i=1, \ldots, n$ and $x \in \mathbb{R}^{n}$ the homogeneous norm can be defined, for example, as follows

$$
|x|_{r}=\left(\sum_{i=1}^{n}\left|x_{i}\right|^{\rho / r_{i}}\right)^{1 / \rho}, \rho \geq r_{\max } .
$$

For all $x \in \mathbb{R}^{n}$, its Euclidean norm $\|x\|$ is related with the homogeneous one:

$$
\underline{\sigma}_{r}\left(|x|_{r}\right) \leq\|x\| \leq \bar{\sigma}_{r}\left(|x|_{r}\right)
$$

for some $\underline{\sigma}_{r}, \bar{\sigma}_{r} \in \mathcal{K}_{\infty}$ [24]. The homogeneous norm has an important property that is $\left|\Lambda_{r}(\lambda) x\right|_{r}=$ $\lambda|x|_{r}$ for all $x \in \mathbb{R}^{n}$.

An advantage of homogeneous systems described by ordinary differential equations is that any its solution can be obtained from another solution under the dilation and a suitable time re-parametrization [11]. A similar property is satisfied for homogeneous time-delay dynamics with scaled delay value [23]. 


\subsection{Inequalities}

The Young's inequality claims that for any $\mathfrak{a}, \mathfrak{b} \in \mathbb{R}_{+}$and $\gamma>0, \delta>0$ [26]:

$$
\mathfrak{a}^{\gamma} \mathfrak{b}^{\delta} \leq \frac{1}{p} \mathfrak{a}^{\gamma p}+\frac{p-1}{p} \mathfrak{b}^{\frac{\delta p}{p-1}}
$$

for any $p>1$, while Hölder's inequality for any $f, g: I \rightarrow \mathbb{R}$ with $I \subset \mathbb{R}$ ensures that [26]:

$$
\int_{I}|f(s) g(s)| d s \leq\left(\int_{I}|f(s)|^{p} d s\right)^{\frac{1}{p}}\left(\int_{I}|g(s)|^{\frac{p}{p-1}} d s\right)^{\frac{p-1}{p}}
$$

for any $p>1$.

Using the properties of homogeneous functions the following result can be obtained [17]:

Lemma 1. Let $\mathfrak{a}, \mathfrak{b} \in \mathbb{R}_{+}$and $\ell>0, \alpha>0, \beta>0, \gamma>0, \delta>0$ be given, then

$$
\mathfrak{a}^{\alpha}+\mathfrak{b}^{\beta}-\ell \mathfrak{a}^{\gamma} \mathfrak{b}^{\delta} \geq 0
$$

provided that
1) $\max \left\{\mathfrak{a}^{\alpha}, \mathfrak{b}^{\beta}\right\} \geq \ell^{\frac{1}{1-\frac{\gamma}{\alpha}-\frac{\delta}{\beta}}}$ and $\frac{\gamma}{\alpha}+\frac{\delta}{\beta}<1$,
2) $\max \left\{\mathfrak{a}^{\alpha}, \mathfrak{b}^{\beta}\right\} \leq \ell^{\frac{1}{1-\frac{\gamma}{\alpha}-\frac{\delta}{\beta}}}$ and $\frac{\gamma}{\alpha}+\frac{\delta}{\beta}>1$.

\section{Problem Formulation}

Consider two switched time-delay systems with synchronous

$$
\dot{x}(t)=F^{(\sigma(t))}(x(t))+G^{(\sigma(t))}(x(t-\tau))
$$

and asynchronous

$$
\dot{x}(t)=F^{(\sigma(t))}(x(t))+G^{(\sigma(t-\tau))}(x(t-\tau))
$$

switching, where $x(t) \in \mathbb{R}^{n}, \sigma:[-\tau,+\infty) \mapsto\{1, \ldots, N\}$ is a piecewise constant rightcontinuous function defining switching law, integer $N>0$ determines the number of subsystems in (1) and (2); the vector functions $F^{(s)}(x)$ and $G^{(s)}(x)$ are continuous for $x \in \mathbb{R}^{n}, s=1, \ldots, N$; $\tau$ is a constant positive delay. Let initial functions for (1) and (2) be chosen from the space $C\left([-\tau, 0], \mathbb{R}^{n}\right)$, then under introduced hypotheses the respective solution of (1) and (2) is defined in forward time at least locally. Denote by $x_{t}$ the restriction of a solution $x(t)$ to the segment $[t-\tau, t]$, i.e., $x_{t}: \xi \mapsto x(t+\xi), \xi \in[-\tau, 0]$.

In the present paper, we will assume that $\sigma(t)$ is a non-Zeno switching signal [40], i.e., on every bounded time interval the set of its instants of discontinuity is finite. Define these switching instants by $\eta_{i}, i=1,2, \ldots$, which satisfy the property $0<\eta_{1}<\eta_{2}<\ldots$ under the absence of Zeno behavior.

The terms $G^{(\sigma(t))}(x(t-\tau))$ and $G^{(\sigma(t-\tau))}(x(t-\tau))$ in (1) and (2), respectively, may be considered as representing the retarded control inputs, and the delay $\tau$ may be a result of a time lag needed for the state to become available for the controller. To clarify this observation, consider an open-loop switched system with a delayed input:

$$
\dot{x}(t)=F^{(\sigma(t))}(x(t))+B_{\sigma(t)} u(t-\tau)
$$

under a feedback control

$$
u(t)=H(x(t)),
$$


where $B_{i}$ is a matrix of appropriate dimension, and $H$ is a function such that $G^{(i)}(x)=B_{i} H(x)$ satisfies the required conditions, $i \in\{1, \ldots, N\}$, and all other variables have the same meaning as before. Hence, (1) can be interpreted as a switched system closed by a common feedback for all subsystems. To introduce the system (2), consider a slightly modified open-loop dynamics:

$$
\dot{x}(t)=F^{(\sigma(t))}(x(t))+B u(t-\tau)
$$

under a feedback control

$$
u(t)=H^{(\sigma(t))}(x(t)),
$$

where $B$ is a common control gain of proper dimension, and $H^{(i)}$ is a function such that $G^{(i)}(x)=B H^{(i)}(x)$ satisfies the required conditions, $i \in\{1, \ldots, N\}$. Therefore, in such a case the switched system is regulated by control adapted to each subsystem leading to an asynchronous commutation. Therefore, in (1) it is supposed that switching between system and control modes is synchronous, i.e., the controller is common for all nodes and does not need an information about the current mode, while in (2) we have asynchronous switching since the control is mode-dependent. It is known (see, for instance, [42, 58]), that asynchronous switching occurs in a wide class of practical problems, where there exists a communication channel between the information about the switching signal of the plant and that of the controller, or where some time is necessary to identify an active subsystem and apply the matched controller.

Assumption 1. For all $s=1, \ldots, N$ the functions $F^{(s)}(x)$ and $G^{(s)}(x)$ are r-homogeneous of the degree $\nu$ with respect to weights $\mathbf{r}=\left(r_{1}, \ldots, r_{n}\right)$, where $r_{i}>0, \nu+r_{i}>0, i=1, \ldots, n$.

Under Assumption 1, the systems (1) and (2) admit zero solutions. Our objective is to derive the conditions of delay-independent stability at the origin for these systems. Moreover, we will look for delay-independent uniform ultimate boundedness conditions for (1) and (2). To this end, we will use both Lyapunov-Razumikhin and Lyapunov-Krasovskii approaches with a comparison of the effectiveness of these methods.

\section{Application of the Lyapunov-Razumikhin Approach}

First, consider the system (1) and the associated family of the delay-free subsystems:

$$
\dot{x}(t)=F^{(s)}(x(t))+G^{(s)}(x(t)), \quad s=1, \ldots, N .
$$

Assumption 2. The family (3) admits a common Lyapunov function $V(x)$ with the following properties:

(i) $V(x)$ is continuously differentiable for $x \in \mathbb{R}^{n}$;

(ii) $V(x)$ is positive definite;

(iii) $V(x)$ is $\mathbf{r}$-homogeneous of the degree $\mu$ with respect to weights $\mathbf{r}=\left(r_{1}, \ldots, r_{n}\right)$, where $\mu>r_{i}, i=1, \ldots, n$;

(iv) the functions $(\partial V(x) / \partial x)^{\top}\left(F^{(s)}(x)+G^{(s)}(x)\right), s=1, \ldots, N$, are negative definite.

Note that under Assumption 1 each subsystem in (3) is $\mathbf{r}$-homogeneous for all $s=1, \ldots, N$, and if it is asymptotically stable, then there is a Lyapunov function as it is given in Assumption 2 [60, 48]. The only restriction is the existence of the common function $V$ for all subsystems in $(3)$.

Theorem 1. Let assumptions 1 and 2 be fulfilled, and consider (1) with any admissible switching law $\sigma$ and any $\tau>0$ :

- if $\nu>0$, then the zero solution is asymptotically stable;

- if $\nu<0$, then solutions are uniformly ultimately bounded. 
Proof. Let a Lyapunov function $V(x)$ possess the properties specified in Assumption 2. Consider its derivative along the solutions of (1). We obtain

$$
\begin{gathered}
\dot{V}=\left(\frac{\partial V(x(t))}{\partial x}\right)^{\top}\left(F^{(\sigma(t))}(x(t))+G^{(\sigma(t))}(x(t-\tau))\right) \\
=\left(\frac{\partial V(x(t))}{\partial x}\right)^{\top}\left(F^{(\sigma(t))}(x(t))+G^{(\sigma(t))}(x(t))\right)+\left(\frac{\partial V(x(t))}{\partial x}\right)^{\top}\left(G^{(\sigma(t))}(x(t-\tau))-G^{(\sigma(t))}(x(t))\right) .
\end{gathered}
$$

Using Assumption 2 and properties of homogeneous functions [11, we arrive at the inequality

$$
\dot{V} \leq-c_{1}|x(t)|_{r}^{\mu+\nu}+c_{2} \sum_{i=1}^{n}|x(t)|_{r}^{\mu-r_{i}}\left|G_{i}^{(\sigma(t))}(x(t-\tau))-G_{i}^{(\sigma(t))}(x(t))\right|,
$$

where $c_{1}, c_{2}$ are positive constants and $G_{i}^{(\sigma(t))}(x)$ are components of the vectors $G^{(\sigma(t))}(x)$.

If $x(t) \neq 0$, then

$$
\begin{gathered}
\left|G_{i}^{(s)}(x(t-\tau))-G_{i}^{(s)}(x(t))\right|=|x(t)|_{r}^{\nu+r_{i}}\left|G_{i}^{(s)}\left(\Lambda^{-1}\left(|x(t)|_{r}\right) x(t-\tau)\right)-G_{i}^{(s)}\left(\Lambda^{-1}\left(|x(t)|_{r}\right) x(t)\right)\right| \\
=|x(t)|_{r}^{\nu+r_{i}}\left|G_{i}^{(s)}\left(z(t)+\Lambda^{-1}\left(|x(t)|_{r}\right)(x(t-\tau)-x(t))\right)-G_{i}^{(s)}(z(t))\right|
\end{gathered}
$$

for $i=1, \ldots, n, s=1, \ldots, N$, where $z(t)=\Lambda^{-1}\left(|x(t)|_{r}\right) x(t)$. Let us note that $|z(t)|_{r}=1$.

We have

$$
\left|x_{i}(t)-x_{i}(t-\tau)\right| \leq\left|x_{i}(t)-x_{i}\left(t-t_{1}\right)\right|+\left|x_{i}\left(t-t_{1}\right)-x_{i}\left(t-t_{2}\right)\right|+\ldots+\left|x_{i}\left(t-t_{m}\right)-x_{i}(t-\tau)\right|
$$

for $i=1, \ldots, n$ and some $m \geq 0$, which corresponds to the number of commutations on the interval $[t-\tau, t]$. Denote $t_{0}=0, t_{m+1}=\tau$. Then on each interval by applying the Mean Value Theorem, we obtain

$$
\left|x_{i}\left(t-t_{j}\right)-x_{i}\left(t-t_{j+1}\right)\right|=\left|t_{j}-t_{j+1}\right|\left|F_{i}^{\left(\sigma\left(t-\theta_{i j}\right)\right)}\left(x\left(t-\theta_{i j}\right)\right)+G_{i}^{\left(\sigma\left(t-\theta_{i j}\right)\right)}\left(x\left(t-\tau-\theta_{i j}\right)\right)\right|
$$

for $i=1, \ldots, n, j=0, \ldots, m$, where $t_{j+1}<\theta_{i j}<t_{j}$ and $F_{i}^{(\sigma(t))}(x)$ are components of the vectors $F^{(\sigma(t))}(x)$. Hence,

$$
|x(t)|_{r}^{-r_{i}}\left|x_{i}(t-\tau)-x_{i}(t)\right| \leq \tau c_{3}|x(t)|_{r}^{-r_{i}} \sup _{\xi \in[t-2 \tau, t]}|x(\xi)|_{r}^{\nu+r_{i}}
$$

for $i=1, \ldots, n$, where $c_{3}=$ const $>0$.

Let for a solution $x(t)$ of (1) the Razumikhin condition $V(x(\xi)) \leq \rho V(x(t))$ be fulfilled for $\xi \in[t-2 \tau, t]$ with $\rho>1$. By homogeneity of $V$ we have:

$$
a_{1}|x(\xi)|_{r}^{\mu} \leq V(x(\xi)) \leq \rho V(x(t)) \leq \rho a_{2}|x(t)|_{r}^{\mu},
$$

where $a_{1}>0, a_{2}>0$. Hence, there exists a positive number $\hat{a}$ such that

$$
|x(\xi)|_{r} \leq \hat{a}|x(t)|_{r}
$$

for $\xi \in[t-2 \tau, t]$. Using estimates (4) and (5), it is easy to verify that

$$
\left\|\Lambda^{-1}\left(|x(t)|_{r}\right)(x(t-\tau)-x(t))\right\| \leq b|x(t)|_{r}^{\nu}, \quad b=\text { const }>0 .
$$


The functions $G^{(1)}(x), \ldots, G^{(N)}(x)$ are assumed to be continuous, hence, by Heine-Cantor Theorem, they are uniformly continuous on any compact set. Therefore, if $\nu>0$, then one can choose $\delta_{1}>0$ such that

$$
\left|G_{i}^{(s)}\left(z(t)+\Lambda^{-1}\left(|x(t)|_{r}\right)(x(t-\tau)-x(t))\right)-G_{i}^{(s)}(z(t))\right|<\frac{c_{1}}{2 n c_{2}}, \quad i=1, \ldots, n, \quad s=1, \ldots, N,
$$

for $\|x(t)\|<\delta_{1}$, whereas if $\nu<0$, then there exists $\delta_{2}>0$ such that the inequalities (6) hold for $\|x(t)\|>\delta_{2}$ (consequently, the uniform continuity is used on the set $\left\{x \in \mathbb{R}^{n}:\|x\| \leq\right.$ $\left.\left.1+b \max ^{\nu}\left\{\underline{\sigma}_{r}^{-1}\left(\delta_{1}\right), \bar{\sigma}_{r}^{-1}\left(\delta_{2}\right)\right\}\right\}\right)$. As a result, we arrive at the estimate

$$
\dot{V} \leq-\frac{1}{2} c_{1}|x(t)|_{r}^{\mu+\nu}
$$

The application of theorems 4.2 and 4.3 from [29] completes the proof.

The obtained result demonstrates that for the selected class of nonlinear systems, the stability of each subsystem with a common Lyapunov function in the delay-free case leads to independent of delay stability (of different kinds depending on degree of the homogeneity) under arbitrary synchronous commutation.

Next, consider the case of asynchronous switching. Construct the auxiliary family of delayfree subsystems:

$$
\dot{x}(t)=F^{(s)}(x(t))+G^{(l)}(x(t)), \quad s, l=1, \ldots, N .
$$

Assumption 3. The family (7) admits a common Lyapunov function $\widetilde{V}(x)$ with the following properties:

(i) $\widetilde{V}(x)$ is continuously differentiable for $x \in \mathbb{R}^{n}$;

(ii) $\widetilde{V}(x)$ is positive definite;

(iii) $\widetilde{V}(x)$ is $\mathbf{r}$-homogeneous of the degree $\mu$ with respect to weights $\mathbf{r}=\left(r_{1}, \ldots, r_{n}\right)$, where $\mu>r_{i}, i=1, \ldots, n$;

(iv) the functions $(\partial \widetilde{V}(x) / \partial x)^{\top}\left(F^{(s)}(x)+G^{(l)}(x)\right), s, l=1, \ldots, N$, are negative definite.

Contrarily Assumption 2, in this hypothesis we ask that any possible combinations of own dynamics $F^{(s)}(x(t))$ and control gains $G^{(l)}(x(t))$ with $s, l=1, \ldots, N$ admit a common Lyapunov function $\widetilde{V}(x)$ in the delay-free case.

Theorem 2. Let assumptions 1 and 3 be fulfilled, and consider (2) with any admissible switching law $\sigma$ and any $\tau>0$ :

- if $\nu>0$, then the zero solution is asymptotically stable;

- if $\nu<0$, then solutions are uniformly ultimately bounded.

Proof. Consider a Lyapunov function $\widetilde{V}(x)$ satisfying the conditions of Assumption 3. Differentiating $\widetilde{V}(x)$ along the solutions of (2), we obtain

$$
\begin{aligned}
\dot{\tilde{V}} & =\left(\frac{\partial \widetilde{V}(x(t))}{\partial x}\right)^{\top}\left(F^{(\sigma(t))}(x(t))+G^{(\sigma(t-\tau))}(x(t-\tau))\right) \\
& =\left(\frac{\partial \widetilde{V}(x(t))}{\partial x}\right)^{\top}\left(F^{(\sigma(t))}(x(t))+G^{(\sigma(t-\tau))}(x(t))\right) \\
& +\left(\frac{\partial \widetilde{V}(x(t))}{\partial x}\right)^{\top}\left(G^{(\sigma(t-\tau))}(x(t-\tau))-G^{(\sigma(t-\tau))}(x(t))\right)
\end{aligned}
$$




$$
\leq-\tilde{c}_{1}|x(t)|_{r}^{\mu+\nu}+\tilde{c}_{2} \sum_{i=1}^{n}|x(t)|_{r}^{\mu-r_{i}}\left|G_{i}^{(\sigma(t-\tau))}(x(t-\tau))-G_{i}^{(\sigma(t-\tau))}(x(t))\right|,
$$

where $\tilde{c}_{1}, \tilde{c}_{2}$ are positive constants. The subsequent proof is a similar to that of Theorem 1.

As we can conclude, the results obtained for synchronous and asynchronous switching are the same, but in the latter case more restrictive conditions are imposed (Assumption 3 implies Assumption 2).

Remark 1. To construct Lyapunov functions possessing the properties specified in assumptions 2 and 3, the approach proposed in [2] may be useful. It is worth noting that the time-varying delays can be treated using Lyapunov-Razumikhin approach as in [6], and that in this work the Lipschitz continuity of the right-hand sides of (1) or (2) used in [6] is relaxed to a merely continuity.

\section{Application of the Lyapunov-Krasovskii Approach}

Next, determine conditions under which there exist Lyapunov-Krasovskii functionals guaranteeing delay-independent asymptotic stability or uniform ultimate boundedness for the systems (1) and (2) under arbitrary switching. For this purpose we will use a recent result [17], which suggests a generic structure of Lyapunov-Krasovskii functional for a homogeneous system that is asymptotically stable in the delay-free case.

Assumption 4. The family (7) admits a common Lyapunov function $\widetilde{V}(x)$ that is twice continuously differentiable for $x \in \mathbb{R}^{n}$ and possesses the properties (ii)-(iv) from Assumption 3.

Using the approach developed in [17], construct a Lyapunov-Krasovskii functional candidate

$$
\widetilde{W}\left(t, x_{t}\right)=\widetilde{V}(x(t))+\left(\frac{\partial \widetilde{V}(x(t))}{\partial x}\right)^{\top} \int_{t-\tau}^{t} G^{(\sigma(\zeta+\tau))}(x(\zeta)) d \zeta+\int_{t-\tau}^{t}(\beta+\gamma(\zeta-t+\tau))|x(\zeta)|_{r}^{\lambda} d \zeta
$$

where $\widetilde{V}(x)$ is a Lyapunov function with the properties specified in Assumption $4, \beta, \gamma, \lambda$ are positive parameters.

Theorem 3. Let assumptions 1 and 4 be fulfilled, and consider (1) for any admissible switching law $\sigma$ and any $\tau>0$. Then there exists a Lyapunov-Krasovskii functional of the form (8) ensuring:

- if $\nu>0$, the asymptotic stability of the zero solution;

- if $\nu<0$, the uniform ultimate boundedness of solutions.

Proof. Consider the Lyapunov-Krasovskii functional (8) and its derivative along the solutions of (1). Using Assumption 4 and properties of homogeneous functions, we obtain

$$
\begin{gathered}
\tilde{c}_{1}|x(t)|_{r}^{\mu}-\tilde{c}_{3} \sum_{i=1}^{n}|x(t)|_{r}^{\mu-r_{i}} \int_{t-\tau}^{t}|x(\zeta)|_{r}^{\nu+r_{i}} d \zeta+\beta \int_{t-\tau}^{t}|x(\zeta)|_{r}^{\lambda} d \zeta \leq \widetilde{W}\left(t, x_{t}\right) \\
\leq \tilde{c}_{2}|x(t)|_{r}^{\mu}+\tilde{c}_{3} \sum_{i=1}^{n}|x(t)|_{r}^{\mu-r_{i}} \int_{t-\tau}^{t}|x(\zeta)|_{r}^{\nu+r_{i}} d \zeta+(\beta+\gamma \tau) \int_{t-\tau}^{t}|x(\zeta)|_{r}^{\lambda} d \zeta \\
\dot{\widetilde{W}}=\left(\frac{\partial \widetilde{V}(x(t))}{\partial x}\right)^{\top}\left(F^{(\sigma(t))}(x(t))+G^{(\sigma(t))}(x(t-\tau))\right)
\end{gathered}
$$




$$
\begin{gathered}
+\left(\frac{\partial \widetilde{V}(x(t))}{\partial x}\right)^{\top}\left(G^{(\sigma(t+\tau))}(x(t))-G^{(\sigma(t))}(x(t-\tau))\right) \\
+(\beta+\gamma \tau)|x(t)|_{r}^{\lambda}-\beta|x(t-\tau)|_{r}^{\lambda}-\gamma \int_{t-\tau}^{t}|x(\zeta)|_{r}^{\lambda} d \zeta \\
+\sum_{i, j=1}^{n} \frac{\partial^{2} \widetilde{V}(x(t))}{\partial x_{i} \partial x_{j}}\left(F_{j}^{(\sigma(t))}(x(t))+G_{j}^{(\sigma(t))}(x(t-\tau))\right) \int_{t-\tau}^{t} G_{i}^{(\sigma(\zeta+\tau))}(x(\zeta)) d \zeta \\
\leq-\tilde{c}_{4}|x(t)|_{r}^{\mu+\nu}+\tilde{c}_{5} \sum_{i, j=1}^{n}|x(t)|_{r}^{\mu-r_{i}-r_{j}}\left(|x(t)|_{r}^{\nu+r_{j}}+|x(t-\tau)|_{r}^{\nu+r_{j}}\right) \int_{t-\tau}^{t}|x(\zeta)|_{r}^{\nu+r_{i}} d \zeta \\
+(\beta+\gamma \tau)|x(t)|_{r}^{\lambda}-\beta|x(t-\tau)|_{r}^{\lambda}-\gamma \int_{t-\tau}^{t}|x(\zeta)|_{r}^{\lambda} d \zeta,
\end{gathered}
$$

where $\tilde{c}_{1}, \ldots, \tilde{c}_{5}$ are positive constants.

Let $\lambda=\mu+\nu$ and $\beta+\gamma \tau<\tilde{c}_{4}$. Using Lemma 1, Young's and Hölder's inequalities, it can be verified that

$$
\begin{gathered}
|x(t)|_{r}^{\mu-r_{i}} \int_{t-\tau}^{t}|x(\zeta)|_{r}^{\nu+r_{i}} d \zeta \leq \tilde{c}_{6}|x(t)|_{r}^{\mu-r_{i}}\left(\int_{t-\tau}^{t}|x(\zeta)|_{r}^{\nu+r_{i}} d \zeta\right)^{\frac{\nu+r_{i}}{\nu+\mu}} \\
\leq \tilde{c}_{7}\left(|x(t)|_{r}^{\mu}+\int_{t-\tau}^{t}|x(\zeta)|_{r}^{\nu+\mu} d \zeta\right)^{\alpha_{i}}, \\
|x(t)|_{r}^{\mu-r_{i}+\nu} \int_{t-\tau}^{t}|x(\zeta)|_{r}^{\nu+r_{i}} d \zeta \leq \tilde{c}_{8}|x(t)|_{r}^{\mu-r_{i}+\nu}\left(\int_{t-\tau}^{t}|x(\zeta)|_{r}^{\nu+r_{i}} d \zeta\right)^{\frac{\nu+r_{i}}{\nu+\mu}} \\
\leq \tilde{c}_{9}\left(|x(t)|_{r}^{\mu+\nu}+\int_{t-\tau}^{t}|x(\zeta)|_{r}^{\nu+\mu} d \zeta\right)^{\omega}
\end{gathered}
$$

for $i=1, \ldots, n$, where $\tilde{c}_{l}>0$ for $l=6, \ldots, 9, \alpha_{i}=\left(\mu(2 \nu+\mu)-r_{i} \nu\right) /(\mu(\nu+\mu))$ and $\omega=$ $1+\nu /(\mu+\nu)$.

In the same vein:

$$
\begin{gathered}
|x(t)|_{r}^{\mu-r_{i}-r_{j}}|x(t-\tau)|_{r}^{\nu+r_{j}} \int_{t-\tau}^{t}|x(\zeta)|_{r}^{\nu+r_{i}} d \zeta \\
\leq \tilde{c}_{10}\left(|x(t)|_{r}^{\mu+\nu}+|x(t-\tau)|_{r}^{\mu+\nu}+\int_{t-\tau}^{t}|x(\zeta)|_{r}^{\mu+\nu}\right)^{\omega}, \quad i, j=1, \ldots, n,
\end{gathered}
$$

for $\nu<0$,

$$
\begin{gathered}
|x(t)|_{r}^{\mu-r_{i}-r_{j}}|x(t-\tau)|_{r}^{\nu+r_{j}} \int_{t-\tau}^{t}|x(\zeta)|_{r}^{\nu+r_{i}} d \zeta \\
\leq \tilde{c}_{11}\left(|x(t)|_{r}^{\mu+\nu}+|x(t-\tau)|_{r}^{\mu+\nu}+\int_{t-\tau}^{t}|x(\zeta)|_{r}^{\mu+\nu}\right)\left(\int_{t-\tau}^{t}|x(\zeta)|_{r}^{\mu+\nu}\right)^{\frac{\nu}{\mu+\nu}}, \quad i, j=1, \ldots, n,
\end{gathered}
$$

for $\nu>0$, where $\tilde{c}_{10}>0, \tilde{c}_{11}>0$.

As a result, we arrive at the estimates

$$
\min \left\{\tilde{c}_{1} ; \beta\right\}\left(|x(t)|_{r}^{\mu}+\int_{t-\tau}^{t}|x(\zeta)|_{r}^{\nu+\mu} d \zeta\right)-\tilde{c}_{3} \tilde{c}_{7} \sum_{i=1}^{n}\left(|x(t)|_{r}^{\mu}+\int_{t-\tau}^{t}|x(\zeta)|_{r}^{\nu+\mu} d \zeta\right)^{\alpha_{i}} \leq \widetilde{W}\left(t, x_{t}\right)
$$




$$
\leq \max \left\{\tilde{c}_{1} ; \beta\right\}\left(|x(t)|_{r}^{\mu}+\int_{t-\tau}^{t}|x(\zeta)|_{r}^{\nu+\mu} d \zeta\right)+\tilde{c}_{3} \tilde{c}_{7} \sum_{i=1}^{n}\left(|x(t)|_{r}^{\mu}+\int_{t-\tau}^{t}|x(\zeta)|_{r}^{\nu+\mu} d \zeta\right)^{\alpha_{i}}
$$

for $\nu \neq 0$, and

$$
\begin{gathered}
\dot{\widetilde{W}} \leq-h\left(|x(t)|_{r}^{\mu+\nu}+|x(t-\tau)|_{r}^{\mu+\nu}+\int_{t-\tau}^{t}|x(\zeta)|_{r}^{\mu+\nu}\right) \\
+\tilde{c}_{12}\left(|x(t)|_{r}^{\mu+\nu}+|x(t-\tau)|_{r}^{\mu+\nu}+\int_{t-\tau}^{t}|x(\zeta)|_{r}^{\mu+\nu}\right)^{\omega} \\
+\tilde{c}_{13}\left(|x(t)|_{r}^{\mu+\nu}+\int_{t-\tau}^{t}|x(\zeta)|_{r}^{\mu+\nu}\right)^{\omega}
\end{gathered}
$$

for $\nu<0$,

$$
\begin{gathered}
\dot{\widetilde{W}} \leq-h\left(|x(t)|_{r}^{\mu+\nu}+|x(t-\tau)|_{r}^{\mu+\nu}+\int_{t-\tau}^{t}|x(\zeta)|_{r}^{\mu+\nu}\right) \\
+\tilde{c}_{14}\left(|x(t)|_{r}^{\mu+\nu}+\right. \\
\left.|x(t-\tau)|_{r}^{\mu+\nu}+\int_{t-\tau}^{t}|x(\zeta)|_{r}^{\mu+\nu}\right)\left(\int_{t-\tau}^{t}|x(\zeta)|_{r}^{\mu+\nu}\right)^{\frac{\nu}{\mu+\nu}} \\
+\tilde{c}_{13}\left(|x(t)|_{r}^{\mu+\nu}+\int_{t-\tau}^{t}|x(\zeta)|_{r}^{\mu+\nu}\right)^{\omega}
\end{gathered}
$$

for $\nu>0$, where $h=\min \left\{\tilde{c}_{4}-\beta-\gamma \tau ; \beta ; \gamma\right\}$ and $\tilde{c}_{12}, \tilde{c}_{13}, \tilde{c}_{14}$ are positive constants.

It should be noted that $\alpha_{i}>1, \omega>1$ for $\nu>0$, and $0<\alpha_{i}<1,0<\omega<1$ for $\nu<0$. Therefore, one can choose positive numbers $\delta_{1}, \delta_{2}$ such that if $\nu<0$, then the inequalities

$$
\begin{gathered}
\frac{1}{2} \min \left\{\tilde{c}_{1} ; \beta\right\}\left(|x(t)|_{r}^{\mu}+\int_{t-\tau}^{t}|x(\zeta)|_{r}^{\nu+\mu} d \zeta\right) \leq \widetilde{W}\left(t, x_{t}\right) \\
\leq 2 \max \left\{\tilde{c}_{1} ; \beta\right\}\left(|x(t)|_{r}^{\mu}+\int_{t-\tau}^{t}|x(\zeta)|_{r}^{\nu+\mu} d \zeta\right), \\
\dot{\widetilde{W}} \leq-\frac{1}{2} h\left(|x(t)|_{r}^{\mu+\nu}+|x(t-\tau)|_{r}^{\mu+\nu}+\int_{t-\tau}^{t}|x(\zeta)|_{r}^{\mu+\nu}\right)
\end{gathered}
$$

hold for $|x(t)|_{r}^{\mu}+\int_{t-\tau}^{t}|x(\zeta)|_{r}^{\nu+\mu} d \zeta>\delta_{1}$, whereas if $\nu>0$, then the inequalities (9), (10) hold for $|x(t)|_{r}^{\mu}+\int_{t-\tau}^{t}|x(\zeta)|_{r}^{\nu+\mu} d \zeta<\delta_{2}$.

From (9) and (10) it follows (see [17]) that $\dot{\widetilde{W}} \leq-b_{1} \widetilde{W}^{1+\frac{\nu}{\mu}}$ for $\nu<0$ in the domain where $\widetilde{W}>\Delta_{1}$, and $\dot{\vec{W}} \leq-b_{2} \widetilde{W}^{1+\frac{\nu}{\mu}}$ for $\nu>0$ in the domain $\widetilde{W}<\Delta_{2}$ for some $b_{1}, b_{2}, \Delta_{1}, \Delta_{2}>0$. This completes the proof.

Next, construct a Lyapunov-Krasovskii functional for the system (2).

Assumption 5. The family (3) admits a common Lyapunov function $V(x)$ that is twice continuously differentiable for $x \in \mathbb{R}^{n}$ and possesses the properties (ii)-(iv) from Assumption 2.

Consider the Lyapunov-Krasovskii functional candidate

$$
W\left(t, x_{t}\right)=V(x(t))+\left(\frac{\partial V(x(t))}{\partial x}\right)^{\top} \int_{t-\tau}^{t} G^{(\sigma(\zeta))}(x(\zeta)) d \zeta+\int_{t-\tau}^{t}(\beta+\gamma(\zeta-t+\tau))|x(\zeta)|_{r}^{\lambda} d \zeta
$$

where $V(x)$ is a Lyapunov function with the properties specified in Assumption $5, \beta, \gamma, \lambda$ are positive parameters. Note that the only difference between the functionals (8) and (11) consists in the use of delayed values of $G^{(\sigma(\zeta))}$ in the latter.

Theorem 4. Let assumptions 1 and 5 be fulfilled, and consider (2) for any admissible switching law $\sigma$ and any $\tau>0$. Then there exists a Lyapunov-Krasovskii functional of the form (8) ensuring: 
- if $\nu>0$, the asymptotic stability of the zero solution;

- if $\nu<0$, the uniform ultimate boundedness of solutions.

Proof. Differentiating the functional (11) along the solutions of (2), we obtain

$$
\begin{gathered}
\dot{W}=\left(\frac{\partial V(x(t))}{\partial x}\right)^{\top}\left(F^{(\sigma(t))}(x(t))+G^{(\sigma(t-\tau))}(x(t-\tau))\right) \\
+\left(\frac{\partial V(x(t))}{\partial x}\right)^{\top}\left(G^{(\sigma(t))}(x(t))-G^{(\sigma(t-\tau))}(x(t-\tau))\right) \\
+(\beta+\gamma \tau)|x(t)|_{r}^{\lambda}-\beta|x(t-\tau)|_{r}^{\lambda}-\gamma \int_{t-\tau}^{t}|x(\zeta)|_{r}^{\lambda} d \zeta \\
+\sum_{i, j=1}^{n} \frac{\partial^{2} V(x(t))}{\partial x_{i} \partial x_{j}}\left(F_{j}^{(\sigma(t))}(x(t))+G_{j}^{(\sigma(t-\tau))}(x(t-\tau))\right) \int_{t-\tau}^{t} G_{i}^{(\sigma(\zeta))}(x(\zeta)) d \zeta \\
\leq-c_{1}|x(t)|_{r}^{\mu+\nu}+c_{2} \sum_{i, j=1}^{n}|x(t)|_{r}^{\mu-r_{i}-r_{j}}\left(|x(t)|_{r}^{\nu+r_{j}}+|x(t-\tau)|_{r}^{\nu+r_{j}}\right) \int_{t-\tau}^{t}|x(\zeta)|_{r}^{\nu+r_{i}} d \zeta \\
+(\beta+\gamma \tau)|x(t)|_{r}^{\lambda}-\beta|x(t-\tau)|_{r}^{\lambda}-\gamma \int_{t-\tau}^{t}|x(\zeta)|_{r}^{\lambda} d \zeta,
\end{gathered}
$$

where $c_{1}, c_{2}$ are positive constants.

The subsequent proof is a similar to that of Theorem 3 .

It is worth highlighting that contrarily the Lyapunov-Razumikhin method, the application of Lyapunov-Krasovskii approach to the system (2) is less constrained than to (1), and for synchronous/asynchronous switching different functionals (8) or (11) are used. Theorem 4 (Assumption 5) uses the conditions of Assumption 2, while for the system (1) in Theorem 3 (Assumption 4) the restrictions of Assumption 3 are imposed. In other words, here Assumption 4 implies Assumption 5. Therefore, for both systems, (1) and (2), the conditions of Assumption 2 are essential for stability and ultimate boundedness, but for the asynchronous commutation case (2) the delay-free common Lyapunov function has to be twice continuously differentiable.

Note also that in the case of linear systems (homogeneous systems of zero degree), the respective conditions of asymptotic stability obtained by the Lyapunov-Krasovskii approach are even more restrictive [55].

\section{Application of Multiple Lyapunov-Krasovskii Func- tionals}

In this section, the case is considered where neither delay-free family (3) nor that of (7) admits a common homogeneous Lyapunov function. Instead of assumptions $2-5$, we will impose the following restrictions on the systems (1) and (2):

Assumption 6. The switching times $\eta_{1}, \eta_{2}, \ldots$ are given, but we have no information on the order of operation of subsystems.

Assumption 7. The zero solution of every subsystem from the family (3) is asymptotically stable.

Our objective is to prove that, under assumptions 6 and 7, the delay-independent asymptotic stability or uniform ultimate boundedness may be guaranteed for the systems (1) and (2), 
provided the switching law satisfies some additional constraints. These constraints can be derived with the aid of special constructions of multiple Lyapunov-Krasovskii functionals.

It is known (see [11]), that the fulfillment of Assumption 7 implies that, for every $s \in$ $\{1, \ldots, N\}$, there exists a Lyapunov function $V_{s}(x)$ with the following properties:

(i) $V_{s}(x)$ is twice continuously differentiable for $x \in \mathbb{R}^{n}$;

(ii) $V_{s}(x)$ is $\mathbf{r}$-homogeneous of the degree $\mu$ with respect to weights $\mathbf{r}=\left(r_{1}, \ldots, r_{n}\right)$, where $\mu>2 r_{i}, i=1, \ldots, n$

(iii) the estimates

$$
\begin{gathered}
\lambda_{s 1}|x|_{r}^{\mu} \leq V_{s}(x) \leq \lambda_{s 2}|x|_{r}^{\mu} \\
\left(\frac{\partial V_{s}(x)}{\partial x}\right)^{\top}\left(F^{(s)}(x)+G^{(s)}(x)\right) \leq-\lambda_{s 3}|x|_{r}^{\mu+\nu}, \\
\left|\frac{\partial V_{s}(x)}{\partial x_{i}}\right| \leq \omega_{s i}|x|_{r}^{\mu-r_{i}}, \quad i=1, \ldots, n,
\end{gathered}
$$

are valid for $x \in \mathbb{R}^{n}$, where $\lambda_{s j}, \omega_{s i}$ are positive constants, $i=1, \ldots, n, j=1,2,3$.

Construct a family of Lyapunov-Krasovskii functionals

$$
\begin{aligned}
& W_{s}\left(x_{t}\right)=V_{s}(x(t))+\left(\frac{\partial V_{s}(x(t))}{\partial x}\right)^{\top} \int_{t-\tau}^{t} G^{(s)}(x(\zeta)) d \zeta \\
& +\int_{t-\tau}^{t}\left(\beta_{s}+\gamma_{s}(\zeta-t+\tau)\right)|x(\zeta)|_{r}^{\mu+\nu} d \zeta, \quad s=1, \ldots, N,
\end{aligned}
$$

for the system (1), where $\beta_{s}, \gamma_{s}$ are positive parameters.

In a similar way as in the proof of Theorem 3, it can be shown that, for an appropriate choice of $\beta_{s}$ and $\gamma_{s}$, there exist positive numbers $\Delta_{1}, \Delta_{2}, p_{j}, q_{j}, j=1, \ldots, 5$, such that

- if $\nu>0$, then

$$
\begin{gathered}
p_{1}\left(|x(t)|_{r}^{\mu}+\int_{t-\tau}^{t}|x(\zeta)|_{r}^{\nu+\mu} d \zeta\right) \leq W_{s}\left(x_{t}\right) \leq p_{2}\left(|x(t)|_{r}^{\mu}+\int_{t-\tau}^{t}|x(\zeta)|_{r}^{\nu+\mu} d \zeta\right) \\
\dot{W}_{s} \leq-p_{3}\left(|x(t)|_{r}^{\mu+\nu}+\int_{t-\tau}^{t}|x(\zeta)|_{r}^{\mu+\nu} d \zeta\right) \leq-p_{4} W_{s}^{1+\frac{\nu}{\mu}} \\
W_{s}\left(x_{t}\right) \leq p_{5} W_{l}\left(x_{t}\right)
\end{gathered}
$$

for $|x(t)|_{r}^{\mu}+\int_{t-\tau}^{t}|x(\zeta)|_{r}^{\nu+\mu} d \zeta<\Delta_{1}, s, l=1, \ldots, N$;

- if $\nu<0$, then

$$
\begin{gathered}
q_{1}\left(|x(t)|_{r}^{\mu}+\int_{t-\tau}^{t}|x(\zeta)|_{r}^{\nu+\mu} d \zeta\right) \leq W_{s}\left(x_{t}\right) \leq q_{2}\left(|x(t)|_{r}^{\mu}+\int_{t-\tau}^{t}|x(\zeta)|_{r}^{\nu+\mu} d \zeta\right), \\
\dot{W}_{s} \leq-q_{3}\left(|x(t)|_{r}^{\mu+\nu}+\int_{t-\tau}^{t}|x(\zeta)|_{r}^{\mu+\nu} d \zeta\right) \leq-q_{4} W_{s}^{1+\frac{\nu}{\mu}}, \\
W_{s}\left(x_{t}\right) \leq q_{5} W_{l}\left(x_{t}\right) \\
\text { for }|x(t)|_{r}^{\mu}+\int_{t-\tau}^{t}|x(\zeta)|_{r}^{\nu+\mu} d \zeta>\Delta_{2}, s, l=1, \ldots, N .
\end{gathered}
$$

Theorem 5. Let assumptions 1, 6, 7 be fulfilled. Then 
- if $\nu>0$ and

$$
\sum_{k=1}^{m-1} p_{5}^{\frac{\nu(k-m)}{\mu}}\left(\eta_{k+1}-\eta_{k}\right) \rightarrow \infty \text { as } m \rightarrow \infty
$$

then the zero solution of (1) is asymptotically stable for any $\tau>0$;

- if $\nu<0$ and

$$
\sum_{k=1}^{m-1} q_{5}^{\frac{\nu k}{\mu}}\left(\eta_{j+k+1}-\eta_{j+k}\right) \rightarrow \infty \text { as } m \rightarrow \infty
$$

uniformly with respect to $j \in\{0,1, \ldots\}$, then solutions of (1) are uniformly ultimately bounded for any $\tau>0$.

Proof. First, assume that $\nu>0$. Consider a switching law satisfying the condition (18) and the associated system (1). Define a piecewise continuously differentiable Lyapunov-Krasovskii functional as follows: $W\left(t, x_{t}\right)=W_{\sigma(t)}\left(x_{t}\right)$.

Let $t_{0} \geq 0, \bar{t}>t_{0}$, and $x\left(t, \varphi, t_{0}\right)$ be a solution of (1) such that $x\left(t_{0}+\xi, \varphi, t_{0}\right)=\varphi(\xi)$ for $\xi \in[-\tau, 0], \psi(t)<\Delta_{1}$ for $t \in\left[t_{0}, \bar{t}\right]$, where

$$
\psi(t)=\left|x\left(t, \varphi, t_{0}\right)\right|_{r}^{\mu}+\int_{t-\tau}^{t}\left|x\left(\zeta, \varphi, t_{0}\right)\right|_{r}^{\nu+\mu} d \zeta
$$

Denote $w(t)=W\left(t, x_{t}\left(\varphi, t_{0}\right)\right)$ and find $j, m \in\{0,1,2, \ldots\}$ such that $t_{0} \in\left[\eta_{j}, \eta_{j+1}\right), \bar{t} \in$ $\left[\eta_{j+m}, \eta_{j+m+1}\right)$. Subsequently integrating the differential inequalities (13) on the intervals $\left[\eta_{j+m}, \bar{t}\right],\left[\eta_{j+m-1}, \eta_{j+m}\right), \ldots,\left[\eta_{j+1}, \eta_{j+2}\right),\left[t_{0}, \eta_{j+1}\right)$ and using the estimates (12) and (14), we obtain that

$$
\begin{gathered}
p_{1}^{-\frac{\nu}{\mu}} \psi^{-\frac{\nu}{\mu}}(\bar{t}) \geq w^{-\frac{\nu}{\mu}}(\bar{t}) \geq w^{-\frac{\nu}{\mu}}\left(t_{0}\right)+p_{4} \frac{\nu}{\mu}\left(\bar{t}-t_{0}\right) \\
\geq p_{2}^{-\frac{\nu}{\mu}} \psi^{-\frac{\nu}{\mu}}\left(t_{0}\right)+p_{4} \frac{\nu}{\mu}\left(\bar{t}-t_{0}\right) \quad \text { for } m=0 \\
p_{1}^{-\frac{\nu}{\mu}} \psi^{-\frac{\nu}{\mu}}(\bar{t}) \geq w^{-\frac{\nu}{\mu}}(\bar{t}) \geq p_{5}^{-\frac{\nu}{\mu} m} w^{-\frac{\nu}{\mu}}\left(t_{0}\right) \\
+p_{4} \frac{\nu}{\mu}\left(p_{5}^{-\frac{\nu}{\mu} m}\left(\eta_{j+1}-t_{0}\right)+p_{5}^{-\frac{\nu}{\mu}(m-1)}\left(\eta_{j+2}-\eta_{j+1}\right)+\ldots+p_{5}^{-\frac{\nu}{\mu}}\left(\eta_{j+m}-\eta_{j+m-1}\right)+\bar{t}-\eta_{j+m}\right) \\
\geq p_{5}^{-\frac{\nu}{\mu} m} p_{2}^{-\frac{\nu}{\mu}} \psi^{-\frac{\nu}{\mu}}\left(t_{0}\right)+p_{4} \frac{\nu}{\mu}\left(p_{5}^{-\frac{\nu}{\mu}(m-1)}\left(\eta_{j+2}-\eta_{j+1}\right)+\ldots+p_{5}^{-\frac{\nu}{\mu}}\left(\eta_{j+m}-\eta_{j+m-1}\right)\right) \text { for } m>0 .
\end{gathered}
$$

In a similar way as in the proof of Theorem 2 in [2], it can be shown that from the inequalities (21), (22) it follows that the zero solution of (1) is asymptotically stable for any positive delay.

Next, consider the case where $\nu<0$ and a switching signal satisfies the condition (19) uniformly with respect to $j \in\{0,1, \ldots\}$.

As in the first case, define a piecewise continuously differentiable Lyapunov-Krasovskii functional $W\left(t, x_{t}\right)=W_{\sigma(t)}\left(x_{t}\right)$, choose $t_{0} \geq 0, \bar{t}>t_{0}$ and $j, m \in\{0,1,2, \ldots\}$ such that $t_{0} \in\left[\eta_{j}, \eta_{j+1}\right), \bar{t} \in\left[\eta_{j+m}, \eta_{j+m+1}\right)$.

Assume that, for a solution $x\left(t, \varphi, t_{0}\right)$ of $(1)$, the inequality $\psi(t)>\Delta_{2}$ holds for $t \in\left[t_{0}, \bar{t}\right]$, where $\psi(t)$ is defined by the formula $(20)$.

Let $w(t)=W\left(t, x_{t}\left(\varphi, t_{0}\right)\right)$. With the aid of (15)-(17), we obtain that

$$
\begin{gathered}
q_{1}^{-\frac{\nu}{\mu}} \psi^{-\frac{\nu}{\mu}}(\bar{t}) \leq w^{-\frac{\nu}{\mu}}(\bar{t}) \leq w^{-\frac{\nu}{\mu}}\left(t_{0}\right)+q_{4} \frac{\nu}{\mu}\left(\bar{t}-t_{0}\right) \\
\leq q_{2}^{-\frac{\nu}{\mu}} \psi^{-\frac{\nu}{\mu}}\left(t_{0}\right)+q_{4} \frac{\nu}{\mu}\left(\bar{t}-t_{0}\right) \quad \text { for } m=0 ;
\end{gathered}
$$




$$
\begin{gathered}
q_{1}^{-\frac{\nu}{\mu}} \psi^{-\frac{\nu}{\mu}}(\bar{t}) \leq w^{-\frac{\nu}{\mu}}(\bar{t}) \leq q_{5}^{-\frac{\nu}{\mu} m} w^{-\frac{\nu}{\mu}}\left(t_{0}\right) \\
+q_{4} \frac{\nu}{\mu}\left(q_{5}^{-\frac{\nu}{\mu} m}\left(\eta_{j+1}-t_{0}\right)+q_{5}^{-\frac{\nu}{\mu}(m-1)}\left(\eta_{j+2}-\eta_{j+1}\right)+\ldots+q_{5}^{-\frac{\nu}{\mu}}\left(\eta_{j+m}-\eta_{j+m-1}\right)+\bar{t}-\eta_{j+m}\right) \\
\leq q_{5}^{-\frac{\nu}{\mu} m}\left(q_{2}^{-\frac{\nu}{\mu}} \psi^{-\frac{\nu}{\mu}}\left(t_{0}\right)+q_{4} \frac{\nu}{\mu}\left(q_{5}^{\frac{\nu}{\mu}}\left(\eta_{j+2}-\eta_{j+1}\right)+\ldots+q_{5}^{\frac{\nu}{\mu}(m-1)}\left(\eta_{j+m}-\eta_{j+m-1}\right)\right)\right) \text { for } m>0 .
\end{gathered}
$$

Using the estimates (23), (24) and the constraint imposed on the switching law, it is easy to show that, for any $\Delta^{*}>0$, there exists $T>0$ such that if $t_{0} \geq 0,\|\varphi\|_{\tau}<\Delta^{*}$, then $\psi\left(t^{*}\right) \leq \Delta_{2}$ for some $t^{*} \in\left[t_{0}, t_{0}+T\right]$.

Furthermore, one can choose $m^{*} \geq 1$ such that

$$
q_{2}^{-\frac{\nu}{\mu}} \Delta_{2}^{-\frac{\nu}{\mu}}+q_{4} \frac{\nu}{\mu}\left(q_{5}^{\frac{\nu}{\mu}}\left(\eta_{j+2}-\eta_{j+1}\right)+\ldots+q_{5}^{\frac{\nu}{\mu}(m-1)}\left(\eta_{j+m}-\eta_{j+m-1}\right)\right) \leq 0
$$

for all $m \geq m^{*}$ and $j \in\{0,1, \ldots\}$. Hence, if $\psi\left(t^{*}\right)<\Delta_{2}$ for some $t^{*} \geq t_{0}$, then

$$
\psi(t) \leq \frac{q_{2}}{q_{1}} q_{5}^{m^{*}} \Delta_{2}
$$

for $t \geq t^{*}$.

Thus, solutions of (1) are uniformly ultimately bounded with the following estimate of the domain of ultimate boundedness:

$$
|x|_{r} \leq\left(\frac{q_{2}}{q_{1}} q_{5}^{m^{*}} \Delta_{2}\right)^{1 / \mu} .
$$

This completes the proof.

The conditions (18) and (19) introduce restrictions on admissible time between commutations. In the literature, in such a case the concept of (average) dwell time is utilized. Unfortunately, such a kind of constraint can be used for the systems having exponentially stable dynamics (or Lyapunov-Krasovskii functionals), while homogeneous time-delay systems of positive or negative degrees (1)-(2) have a convergence slower than any exponential (see (13) and (16)). Therefore, (18) and (19) are proposed, which technically follow the estimates given in (12)-(17).

Next, consider the case of asynchronous switching. Choose a family of Lyapunov-Krasovskii functionals for (2) as follows:

$$
\begin{aligned}
& \widetilde{W}_{s}\left(t, x_{t}\right)=V_{s}(x(t))+\left(\frac{\partial V_{s}(x(t))}{\partial x}\right)^{\top} \int_{t-\tau}^{t} G^{(\zeta)}(x(\zeta)) d \zeta \\
& +\int_{t-\tau}^{t}\left(\beta_{s}+\gamma_{s}(\zeta-t+\tau)\right)|x(\zeta)|_{r}^{\mu+\nu} d \zeta, \quad s=1, \ldots, N,
\end{aligned}
$$

where $V_{s}(x)$ are homogeneous Lyapunov functions with the properties (i)-(iii) constructed for subsystems from (3), $\beta_{s}, \gamma_{s}$ are positive parameters.

Denote $\widetilde{W}\left(t, x_{t}\right)=\widetilde{W}_{\sigma(t)}\left(t, x_{t}\right)$. Differentiating the functional $\widetilde{W}\left(t, x_{t}\right)$ with respect to the system (2), we obtain

$$
\begin{gathered}
\dot{\widetilde{W}}=\left(\frac{\partial V_{\sigma(t)}(x(t))}{\partial x}\right)^{\top}\left(F^{(\sigma(t))}(x(t))+G^{(\sigma(t))}(x(t))\right) \\
+\left(\beta_{\sigma(t)}+\gamma_{\sigma(t)} \tau\right)|x(t)|_{r}^{\nu+\mu}-\beta_{\sigma(t)}|x(t-\tau)|_{r}^{\nu+\mu}-\gamma_{\sigma(t)} \int_{t-\tau}^{t}|x(\zeta)|_{r}^{\nu+\mu} d \zeta
\end{gathered}
$$




$$
\begin{gathered}
+\sum_{i, j=1}^{n} \frac{\partial^{2} V_{\sigma(t)}(x(t))}{\partial x_{i} \partial x_{j}}\left(F_{j}^{(\sigma(t))}(x(t))+G_{j}^{(\sigma(t-\tau))}(x(t-\tau))\right) \int_{t-\tau}^{t} G_{i}^{(\sigma(\zeta))}(x(\zeta)) d \zeta \\
\leq-\bar{c}_{1}|x(t)|_{r}^{\mu+\nu}+\bar{c}_{2} \sum_{i, j=1}^{n}|x(t)|_{r}^{\mu-r_{i}-r_{j}}\left(|x(t)|_{r}^{\nu+r_{j}}+|x(t-\tau)|_{r}^{\nu+r_{j}}\right) \int_{t-\tau}^{t}|x(\zeta)|_{r}^{\nu+r_{i}} d \zeta \\
+\left(\beta_{\sigma(t)}+\gamma_{\sigma(t)} \tau\right)|x(t)|_{r}^{\nu+\mu}-\beta_{\sigma(t)}|x(t-\tau)|_{r}^{\nu+\mu}-\gamma_{\sigma(t)} \int_{t-\tau}^{t}|x(\zeta)|_{r}^{\nu+\mu} d \zeta
\end{gathered}
$$

for $t \in\left[\eta_{k}, \eta_{k+1}\right), k=0,1, \ldots$, where $\bar{c}_{1}, \bar{c}_{2}$ are positive constants.

Hence, for an appropriate choice of $\beta_{s}$ and $\gamma_{s}$, there exist positive numbers $\tilde{\Delta}_{1}, \tilde{\Delta}_{2}, \tilde{p}_{j}, \tilde{q}_{j}$, $j=1, \ldots, 5$, such that

- if $\nu>0$, then

$$
\begin{gathered}
\tilde{p}_{1}\left(|x(t)|_{r}^{\mu}+\int_{t-\tau}^{t}|x(\zeta)|_{r}^{\nu+\mu} d \zeta\right) \leq \widetilde{W}\left(t, x_{t}\right) \leq \tilde{p}_{2}\left(|x(t)|_{r}^{\mu}+\int_{t-\tau}^{t}|x(\zeta)|_{r}^{\nu+\mu} d \zeta\right), \\
\dot{\widetilde{W}} \leq-\tilde{p}_{3}\left(|x(t)|_{r}^{\mu+\nu}+\int_{t-\tau}^{t}|x(\zeta)|_{r}^{\mu+\nu} d \zeta\right) \leq-\tilde{p}_{4} \widetilde{W}^{1+\frac{\nu}{\mu}} \quad \text { for } \quad t \in\left[\eta_{k}, \eta_{k+1}\right), \quad k=0,1, \ldots, \\
\widetilde{W}_{s}\left(x_{t}\right) \leq \tilde{p}_{5} \widetilde{W}_{l}\left(x_{t}\right), \quad s, l=1, \ldots, N,
\end{gathered}
$$

for $|x(t)|_{r}^{\mu}+\int_{t-\tau}^{t}|x(\zeta)|_{r}^{\nu+\mu} d \zeta<\tilde{\Delta}_{1}$

- if $\nu<0$, then

$$
\begin{aligned}
& \qquad \tilde{q}_{1}\left(|x(t)|_{r}^{\mu}+\int_{t-\tau}^{t}|x(\zeta)|_{r}^{\nu+\mu} d \zeta\right) \leq \widetilde{W}\left(t, x_{t}\right) \leq \tilde{q}_{2}\left(|x(t)|_{r}^{\mu}+\int_{t-\tau}^{t}|x(\zeta)|_{r}^{\nu+\mu} d \zeta\right), \\
& \dot{\widetilde{W}} \leq-\tilde{q}_{3}\left(|x(t)|_{r}^{\mu+\nu}+\int_{t-\tau}^{t}|x(\zeta)|_{r}^{\mu+\nu} d \zeta\right) \leq-\tilde{q}_{4} \widetilde{W}^{1+\frac{\nu}{\mu}} \quad \text { for } \quad t \in\left[\eta_{k}, \eta_{k+1}\right), \quad k=0,1, \ldots, \\
& \qquad \widetilde{W}_{s}\left(x_{t}\right) \leq \tilde{q}_{5} \widetilde{W}_{l}\left(x_{t}\right), \quad s, l=1, \ldots, N, \\
& \text { for }|x(t)|_{r}^{\mu}+\int_{t-\tau}^{t}|x(\zeta)|_{r}^{\nu+\mu} d \zeta>\tilde{\Delta}_{2} .
\end{aligned}
$$

Theorem 6. Let assumptions 1, 6, 7 be fulfilled. Then

- if $\nu>0$ and

$$
\sum_{k=1}^{m-1} \tilde{p}_{5}^{\frac{\nu(k-m)}{\mu}}\left(\eta_{k+1}-\eta_{k}\right) \rightarrow \infty \text { as } m \rightarrow \infty,
$$

then the zero solution of (2) is asymptotically stable for any $\tau>0$;

- if $\nu<0$ and

$$
\sum_{k=1}^{m-1} \tilde{q}_{5}^{\frac{\nu k}{\mu}}\left(\eta_{j+k+1}-\eta_{j+k}\right) \rightarrow \infty \text { as } m \rightarrow \infty
$$

uniformly with respect to $j \in\{0,1, \ldots\}$, then solutions of (2) are uniformly ultimately bounded for any $\tau>0$.

Proof. The proof of the theorem is a similar to that of Theorem 5 . 


\section{Application to the Analysis of Dynamics of Mechani- cal Systems}

In this section, we will show that the results of the present paper (more precisely, the ideas of theorems 3 and 4 from Section 5) can be used for investigation of mechanical systems with switched nonlinear force fields.

Let motions of a mechanical system be described by the equations

$$
A \ddot{q}(t)+B \dot{q}(t)+D^{(\sigma(t))}(q(t))+Q^{(\sigma(t))}(q(t-\tau))=0 .
$$

We will assume that $q(t), \dot{q}(t) \in \mathbb{R}^{n}$ are vectors of generalized coordinates and generalized velocities, respectively; $A$ and $B$ are constant nonsingular matrices; $\sigma:[0,+\infty) \mapsto\{1, \ldots, N\}$ is an admissible switching law; for all $s=1, \ldots, N$ the functions $D^{(s)}(q)$ and $Q^{(s)}(q)$ are continuous for $q \in \mathbb{R}^{n}$ and $\mathbf{r}$-homogeneous of the degree $\nu$ with respect to weights $\mathbf{r}=\left(r_{1}, \ldots, r_{n}\right)$, where $r_{i}>0, \nu+r_{i}>0, i=1, \ldots, n ; \tau$ is a constant positive delay. Hence, velocity forces in (25) are non-switched and linear, while positional ones are switched and homogeneous.

It is known (see, for instance, [14, 43, 27, 38]), that nonlinear restoring forces with realvalued powers are used for modeling a wide class of mechanical systems. Such a type forces may occur due to both strong nonlinear material properties and physical configurations. In addition, power-law characteristics of restoring forces provide smooth approximations of nonsmooth forces [38].

It should be noted that the system (25) admits the zero solution. We will look for conditions ensuring delay-independent asymptotic stability of the zero solution or delay-independent ultimate boundedness of solutions for (25).

According to the approach developed in [4, 3], consider the following two isolated subsystems:

$$
\dot{y}(t)=-A^{-1} B y(t)
$$

and the system (1) with

$$
F^{(\sigma(t))}(x)=-B^{-1} D^{(\sigma(t))}(x), \quad G^{(\sigma(t))}(x)=-B^{-1} Q^{(\sigma(t))}(x) .
$$

Assumption 8. The system (26) is asymptotically stable.

Under this hypothesis and also the previously introduced restrictions for the system (1), the following result can be proven:

Theorem 7. Let functions $F^{(\sigma(t))}(x)$ and $G^{(\sigma(t))}(x)$ be defined by the formula $(27)$ and assumptions 1, 4, 8 be fulfilled. Consider (25) with any admissible switching law $\sigma$ and any $\tau>0$ :

- if

$$
\nu>r_{\max }-r_{\min },
$$

then the zero solution is asymptotically stable;

- if

$$
\nu<r_{\min }-r_{\max }
$$

then solutions are uniformly ultimately bounded.

Proof. The substitution

$$
x(t)=q(t)+B^{-1} A \dot{q}(t), \quad y(t)=\dot{q}(t)
$$


transforms the system (25) to the following one:

$$
\begin{aligned}
\dot{x}(t) & =F^{(\sigma(t))}(x(t))+G^{(\sigma(t))}(x(t-\tau))+B^{-1}\left(D^{(\sigma(t))}(x(t))-D^{(\sigma(t))}\left(x(t)-B^{-1} A y(t)\right)\right) \\
& +B^{-1}\left(Q^{(\sigma(t))}(x(t-\tau))-Q^{(\sigma(t))}\left(x(t-\tau)-B^{-1} A y(t-\tau)\right)\right), \\
\dot{y}(t) & =-A^{-1} B y(t)-A^{-1}\left(D^{(\sigma(t))}\left(x(t)-B^{-1} A y(t)\right)+Q^{(\sigma(t))}\left(x(t-\tau)-B^{-1} A y(t-\tau)\right)\right) .
\end{aligned}
$$

This system may be interpreted as a complex system describing interaction of subsystems (1) and (26).

From Assumption 8 it follows that, for any $\rho>1$, there exists a continuously differentiable for $y \in \mathbb{R}^{n}$ homogeneous of the degree $\rho$ (with respect to the standard dilation) Lyapunov function $v(y)$ such that the estimates

$$
a_{1}\|y\|^{\rho} \leq v(y) \leq a_{2}\|y\|^{\rho}, \quad\left\|\frac{\partial v(y)}{\partial y}\right\| \leq a_{3}\|y\|^{\rho-1}, \quad\left(\frac{\partial v(y)}{\partial y}\right)^{\top} A^{-1} B y \geq a_{4}\|y\|^{\rho}
$$

hold for $y \in \mathbb{R}^{n}$, where $a_{j}>0, j=1, \ldots, 4$.

Construct a Lyapunov-Krasovskii functional candidate for (31) in the form

$$
\begin{gathered}
\widetilde{W}\left(t, x_{t}, y_{t}\right)=\widetilde{V}(x(t))+\left(\frac{\partial \widetilde{V}(x(t))}{\partial x}\right)^{\top} \int_{t-\tau}^{t} G^{(\sigma(\zeta+\tau))}(x(\zeta)) d \zeta \\
+\int_{t-\tau}^{t}(\beta+\gamma(\zeta-t+\tau))|x(\zeta)|_{r}^{\nu+\mu} d \zeta+v(y(t))+\int_{t-\tau}^{t}(\alpha+\varepsilon(\zeta-t+\tau))\|y(\zeta)\|^{\rho} d \zeta,
\end{gathered}
$$

where $\alpha, \varepsilon, \beta, \gamma$ are positive parameters, $\widetilde{V}(x)$ is a Lyapunov function with the properties specified in Assumption 4. Then there exist positive numbers $\tilde{c}_{1}, \tilde{c}_{2}, \tilde{c}_{3}$ such that

$$
\begin{gathered}
\tilde{c}_{1}|x(t)|_{r}^{\mu}-\tilde{c}_{3} \sum_{i=1}^{n}|x(t)|_{r}^{\mu-r_{i}} \int_{t-\tau}^{t}|x(\zeta)|_{r}^{\nu+r_{i}} d \zeta+\beta \int_{t-\tau}^{t}|x(\zeta)|_{r}^{\nu+\mu} d \zeta+a_{1}\|y(t)\|^{\rho}+\alpha \int_{t-\tau}^{t}\|y(\zeta)\|^{\rho} d \zeta \\
\leq \widetilde{W}\left(t, x_{t}, y_{t}\right) \leq \tilde{c}_{2}|x(t)|_{r}^{\mu}+\tilde{c}_{3} \sum_{i=1}^{n}|x(t)|_{r}^{\mu-r_{i}} \int_{t-\tau}^{t}|x(\zeta)|_{r}^{\nu+r_{i}} d \zeta+(\beta+\gamma \tau) \int_{t-\tau}^{t}|x(\zeta)|_{r}^{\nu+\mu} d \zeta \\
+a_{2}\|y(t)\|^{\rho}+(\alpha+\varepsilon \tau) \int_{t-\tau}^{t}\|y(\zeta)\|^{\rho} d \zeta .
\end{gathered}
$$

Differentiating the functional (33) with respect to (31) we obtain

$$
\begin{aligned}
\dot{\widetilde{W}} \leq & -\left(\tilde{c}_{4}-\beta-\gamma \tau\right)|x(t)|_{r}^{\nu+\mu}-\beta|x(t-\tau)|_{r}^{\nu+\mu}-\gamma \int_{t-\tau}^{t}|x(\zeta)|_{r}^{\nu+\mu} d \zeta \\
& -\left(a_{4}-\alpha-\varepsilon \tau\right)\|y(t)\|^{\rho}-\alpha\|y(t-\tau)\|^{\rho}-\varepsilon \int_{t-\tau}^{t}\|y(\zeta)\|^{\rho} d \zeta \\
+ & \tilde{c}_{5} \sum_{i, j=1}^{n}|x(t)|_{r}^{\mu-r_{i}-r_{j}}\left(|x(t)|_{r}^{\nu+r_{j}}+|x(t-\tau)|_{r}^{\nu+r_{j}}\right) \int_{t-\tau}^{t}|x(\zeta)|_{r}^{\nu+r_{i}} d \zeta \\
& +\left(\frac{\partial \widetilde{V}(x(t))}{\partial x}\right)^{\top} B^{-1}\left(D^{(\sigma(t))}(x(t))-D^{(\sigma(t))}\left(x(t)-B^{-1} A y(t)\right)\right)
\end{aligned}
$$




$$
\begin{gathered}
+\left(\frac{\partial \widetilde{V}(x(t))}{\partial x}\right)^{\top} B^{-1}\left(Q^{(\sigma(t))}(x(t-\tau))-Q^{(\sigma(t))}\left(x(t-\tau)-B^{-1} A y(t-\tau)\right)\right) \\
-\left(\frac{\partial v(y(t))}{\partial y}\right)^{\top} A^{-1}\left(D^{(\sigma(t))}\left(x(t)-B^{-1} A y(t)\right)+Q^{(\sigma(t))}\left(x(t-\tau)-B^{-1} A y(t-\tau)\right)\right) \\
+\left(\int_{t-\tau}^{t} G^{(\sigma(\zeta+\tau))}(x(\zeta)) d \zeta\right)^{\top} \frac{\partial^{2} \widetilde{V}(x(t))}{\partial x^{2}} B^{-1}\left(D^{(\sigma(t))}(x(t))-D^{(\sigma(t))}\left(x(t)-B^{-1} A y(t)\right)\right) \\
+\left(\int_{t-\tau}^{t} G^{(\sigma(\zeta+\tau))}(x(\zeta)) d \zeta\right)^{\top} \frac{\partial^{2} \widetilde{V}(x(t))}{\partial x^{2}} B^{-1}\left(Q^{(\sigma(t))}(x(t-\tau))-Q^{(\sigma(t))}\left(x(t-\tau)-B^{-1} A y(t-\tau)\right)\right)
\end{gathered}
$$

where $\tilde{c}_{4}, \tilde{c}_{5}$ are positive constants.

In a similar way as in the proof of Theorem 3, with the aid of Lemma 1, Young's and Hölder's inequalities, it it can be verified that, for an appropriate choice of values of parameters $\alpha, \varepsilon, \beta, \gamma$, there exist positive numbers $\Delta_{1}, \Delta_{2}, d_{j}, e_{j}, j=1, \ldots, 3$, such that

- if (28) and

$$
\frac{\mu+\nu}{\nu+r_{\min }}<\rho<\frac{\mu+\nu}{r_{\max }}
$$

then

$$
\begin{gathered}
d_{1}\left(|x(t)|_{r}^{\mu}+\int_{t-\tau}^{t}|x(\zeta)|_{r}^{\nu+\mu} d \zeta+\|y(t)\|^{\rho}+\int_{t-\tau}^{t}\|y(\zeta)\|^{\rho} d \zeta\right) \\
\leq \widetilde{W}\left(t, x_{t}, y_{t}\right) \leq d_{2}\left(|x(t)|_{r}^{\mu}+\int_{t-\tau}^{t}|x(\zeta)|_{r}^{\nu+\mu} d \zeta+\|y(t)\|^{\rho}+\int_{t-\tau}^{t}\|y(\zeta)\|^{\rho} d \zeta\right), \\
\dot{\widetilde{W}} \leq-d_{3}\left(|x(t)|_{r}^{\mu+\nu}+\int_{t-\tau}^{t}|x(\zeta)|_{r}^{\nu+\mu} d \zeta+\|y(t)\|^{\rho}+\int_{t-\tau}^{t}\|y(\zeta)\|^{\rho} d \zeta\right)
\end{gathered}
$$

for

$$
|x(t)|_{r}^{\mu}+\int_{t-\tau}^{t}|x(\zeta)|_{r}^{\nu+\mu} d \zeta+\|y(t)\|^{\rho}+\int_{t-\tau}^{t}\|y(\zeta)\|^{\rho} d \zeta<\Delta_{1}
$$

- if (29) and

$$
\frac{\mu+\nu}{r_{\min }}<\rho<\frac{\mu+\nu}{\nu+r_{\max }}
$$

then

$$
\begin{gathered}
e_{1}\left(|x(t)|_{r}^{\mu}+\int_{t-\tau}^{t}|x(\zeta)|_{r}^{\nu+\mu} d \zeta+\|y(t)\|^{\rho}+\int_{t-\tau}^{t}\|y(\zeta)\|^{\rho} d \zeta\right) \\
\leq \widetilde{W}\left(t, x_{t}, y_{t}\right) \leq e_{2}\left(|x(t)|_{r}^{\mu}+\int_{t-\tau}^{t}|x(\zeta)|_{r}^{\nu+\mu} d \zeta+\|y(t)\|^{\rho}+\int_{t-\tau}^{t}\|y(\zeta)\|^{\rho} d \zeta\right), \\
\dot{\widetilde{W}} \leq-e_{3}\left(|x(t)|_{r}^{\mu+\nu}+\int_{t-\tau}^{t}|x(\zeta)|_{r}^{\nu+\mu} d \zeta+\|y(t)\|^{\rho}+\int_{t-\tau}^{t}\|y(\zeta)\|^{\rho} d \zeta\right)
\end{gathered}
$$

for

$$
|x(t)|_{r}^{\mu}+\int_{t-\tau}^{t}|x(\zeta)|_{r}^{\nu+\mu} d \zeta+\|y(t)\|^{\rho}+\int_{t-\tau}^{t}\|y(\zeta)\|^{\rho} d \zeta>\Delta_{2}
$$


This completes the proof.

Next, along with the system (25), consider the corresponding mechanical system with asynchronous switching in positional forces:

$$
A \ddot{q}(t)+B \dot{q}(t)+D^{(\sigma(t))}(q(t))+Q^{(\sigma(t-\tau))}(q(t-\tau))=0 .
$$

In this case we suppose that an admissible switching signal $\sigma(t)$ is defined for $t \in[-\tau,+\infty)$.

Theorem 8. Let functions $F^{(\sigma(t))}(x)$ and $G^{(\sigma(t))}(x)$ be defined by the formula (27) and assumptions $1,5,8$ be fulfilled. Consider (34) with any admissible switching law $\sigma$ and any $\tau>0$ :

- if the inequality (28) holds, then the zero solution is asymptotically stable;

- if the inequality (29) holds, then solutions are uniformly ultimately bounded.

Proof. Using the substitution (30), transform (34) to the system

$$
\begin{aligned}
\dot{x}(t) & =F^{(\sigma(t))}(x(t))+G^{(\sigma(t-\tau))}(x(t-\tau))+B^{-1}\left(D^{(\sigma(t))}(x(t))-D^{(\sigma(t))}\left(x(t)-B^{-1} A y(t)\right)\right) \\
& +B^{-1}\left(Q^{(\sigma(t-\tau))}(x(t-\tau))-Q^{(\sigma(t-\tau))}\left(x(t-\tau)-B^{-1} A y(t-\tau)\right)\right), \\
\dot{y}(t) & =-A^{-1} B y(t)-A^{-1}\left(D^{(\sigma(t))}\left(x(t)-B^{-1} A y(t)\right)+Q^{(\sigma(t-\tau))}\left(x(t-\tau)-B^{-1} A y(t-\tau)\right)\right) .
\end{aligned}
$$

Choose a Lyapunov-Krasovskii functional candidate in the form

$$
\begin{gathered}
W\left(t, x_{t}, y_{t}\right)=V(x(t))+\left(\frac{\partial V(x(t))}{\partial x}\right)^{\top} \int_{t-\tau}^{t} G^{(\sigma(\zeta))}(x(\zeta)) d \zeta \\
+\int_{t-\tau}^{t}(\beta+\gamma(\zeta-t+\tau))|x(\zeta)|_{r}^{\nu+\mu} d \zeta+v(y(t))+\int_{t-\tau}^{t}(\alpha+\varepsilon(\zeta-t+\tau))\|y(\zeta)\|^{\rho} d \zeta,
\end{gathered}
$$

where $\alpha, \varepsilon, \beta, \gamma$ are positive parameters, $V(x)$ is a Lyapunov function with the properties specified in Assumption 5, and $v(y)$ is a continuously differentiable for $y \in \mathbb{R}^{n}$ homogeneous of the degree $\rho>1$ (with respect to the standard dilation) Lyapunov function that satisfies the estimates (32).

With the aid of the functional (35), the subsequent proof can be carried out in a similar way as that of Theorem 7 .

Remark 2. The homogeneity of $D^{(\sigma(t))}(x)$ and $Q^{(\sigma(t))}(x)$ implies homogeneity of the functions (27) if the matrices $B^{-1}$ and $\Lambda_{r}(\lambda)$ are commuting, i.e., $B^{-1} \Lambda_{r}(\lambda)=\Lambda_{r}(\lambda) B^{-1}$ for all $\lambda>0$.

Remark 3. For instance, the conditions (28), (29) and Assumption 1 for the functions (27) are fulfilled in the case where $D^{(\sigma(t))}(x)$ and $Q^{(\sigma(t))}(x)$ are homogeneous functions with respect to the standard dilation.

Remark 4. To derive conditions of the asymptotic stability or the ultimate boundedness for (25) and (34), the Lyapunov-Razumikhin approach (Section 4) and the approach based on the application of multiple Lyapunov-Krasovskii functionals (Section 6) can be used, as well. 


\section{Results of a Numerical Simulation}

Consider the system (25) with $n=N=2$ and

$$
\begin{gathered}
A=\left[\begin{array}{ll}
4 & 1 \\
3 & 1
\end{array}\right], B=\left[\begin{array}{cc}
1 & -2 \\
1 & -1
\end{array}\right] \\
D^{(1)}(q)=\left[\begin{array}{c}
2\left(q_{1}^{3}-q_{2}^{3}\right) \\
2 q_{1}^{3}-q_{2}^{3}
\end{array}\right], Q^{(1)}(q)=\left[\begin{array}{c}
4 q_{1}^{2} q_{2}+q_{2}^{2} q_{1} \\
2 q_{1}^{2} q_{2}+q_{2}^{2} q_{1}
\end{array}\right], \\
D^{(2)}(q)=\left[\begin{array}{c}
q_{1}^{3}-6 q_{2}^{3} \\
q_{1}^{3}-3 q_{2}^{3}
\end{array}\right], Q^{(2)}(q)=-\left[\begin{array}{c}
12 q_{1}^{2} q_{2}+3 q_{2}^{2} q_{1} \\
6 q_{1}^{2} q_{2}+3 q_{2}^{2} q_{1}
\end{array}\right],
\end{gathered}
$$

then it is easy to verify that the conditions of theorems 7 and 8 are satisfied for $\mathbf{r}=(1,1)$, $\nu=2$ and

$$
V(x)=\widetilde{V}(x)=x_{1}^{2}+\frac{1}{2} x_{2}^{2}, v(y)=\left(y_{1}+y_{2}\right)^{2}+y_{2}^{2} .
$$

Hence for any $\tau>0$ there is $\Delta>0$ such that the origin is asymptotically stable for the system (25) with the domain of attraction $\left\|q_{0}\right\|_{\tau}+\left\|\dot{q}_{0}\right\|_{\tau} \leq \Delta$.

An example of behavior of position $q(t)$ for synchronous and asynchronous switching are shown in figures 1 and 2 for $\tau=1$ and $\sigma(t)=1.5+0.5 \operatorname{sign}(\sin (\pi t)-\cos (t)+\sin (e t))$.

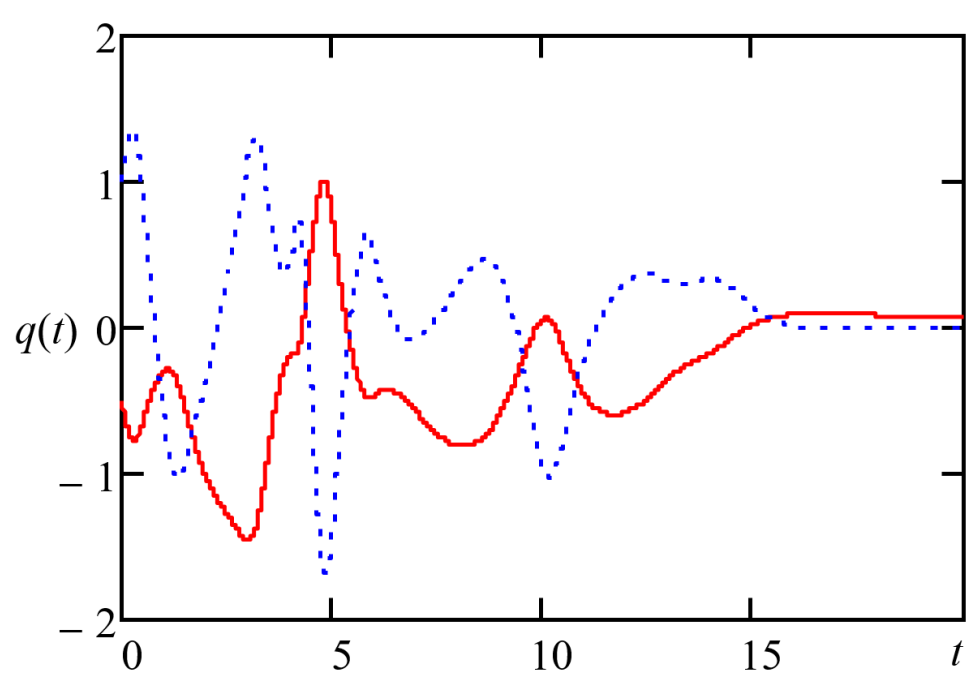

Figure 1: Trajectories of $q(t)$ for synchronous switching

\section{Conclusion}

A switched system was studied, where all subsystems contain time-delays and are homogeneous either of negative or positive degree. It was assumed that these subsystems are asymptotically stable in the delay-free scenario. Two cases were investigated: when the switching signal is delay-free (synchronous switching) or also corrupted by lags (asynchronous switching). In the latter scenario there are terms dependent on a delayed commutation signal, which usually implies that more restrictive conditions have to be imposed on the properties of subsystems and commutation signal to substantiate stability. However, for an arbitrary switching signal by using the common Lyapunov-Krasovskii functional or Lyapunov-Razumikhin function approaches, it was shown that for this class of dynamics the conditions are the same (stability of delayfree subsystems), since one of these approaches is more efficient for synchronous commutation, 


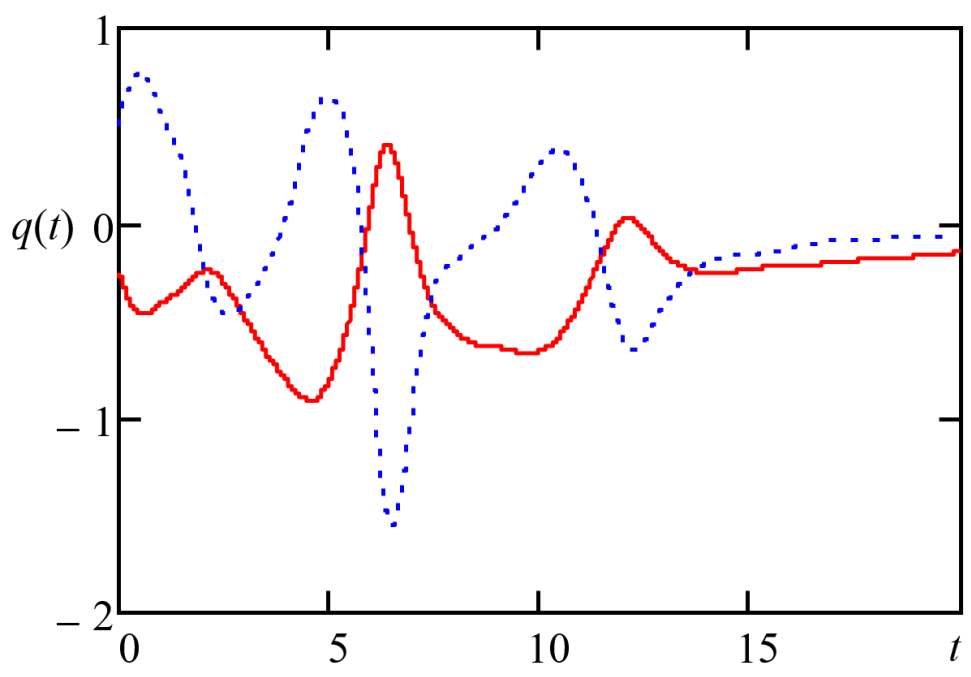

Figure 2: Trajectories of $q(t)$ for asynchronous switching

while another one for the asynchronous switching. The setting with restrictions on the time of commutation between subsystems was explored via multiple Lyapunov-Krasovskii functionals. Since homogeneous systems of non-zero degree are not exponentially stable, the usual dwelltime conditions cannot be applied [18], and other kinds of restrictions were imposed. Stability of a class of mechanical systems with real-valued power restoring forces was analyzed, and an example of simulation was given to illustrate the applicability of the proposed theory.

\section{References}

[1] A. Aleksandrov, A. Fradkov, and A. Semenov. Delayed and switched control of formations on a line segment: Delays and switches do not matter. IEEE Transactions on Automatic Control, 65(2):794-800, 2020.

[2] A.Y. Aleksandrov, A.A. Kosov, and A.V. Platonov. On the asymptotic stability of switched homogeneous systems. Systems and Control Letters, 61(1):127-133, 2012.

[3] A.Yu. Aleksandrov, Y. Chen, A.A. Kosov, and L. Zhang. Stability of hybrid mechanical systems with switching linear force fields. Nonlinear Dynamics and Systems Theory, 11(1):53-64, 2011.

[4] A.Yu. Aleksandrov, A.A. Kosov, and Y. Chen. Stability and stabilization of mechanical systems with switching. Automation and Remote Control, 72(6):1143-1154, 2011.

[5] A.Yu. Aleksandrov and A.P. Zhabko. On the asymptotic stability of solutions of nonlinear systems with delay. Siberian Mathematical Journal, 53(3):393-403, 2012.

[6] A.Yu. Aleksandrov and A.P. Zhabko. Delay-independent stability of homogeneous systems. Applied Mathematics Letters, 34(8):43-50, 2014.

[7] A.Yu. Aleksandrov, A.P. Zhabko, and V.S. Pecherskiy. Complete type functionals for some classes of homogeneous diffrential-difference systems. In Proc. 8th international conference "Modern methods of applied mathematics, control theory and computer technology", pages 5-8, Voronezh, 2015. in Russian. 
[8] V. Andrieu, L. Praly, and A. Astolfi. Homogeneous approximation, recursive observer design, and output feedback. SIAM J. Control Optimization, 47(4):1814-1850, 2008.

[9] F.M. Asl and A.G. Ulsoy. Analytical solution of a system of homogeneous delay differential equations via the Lambert function. In Proc. American Control Conference, pages 24962500, Chicago, 2000.

[10] A. Bacciotti and L. Rosier. Liapunov Functions and Stability in Control Theory, volume 267 of Lecture Notes in Control and Inform. Sci. Springer, Berlin, 2001.

[11] E. Bernuau, D. Efimov, W. Perruquetti, and A. Polyakov. On homogeneity and its application in sliding mode. Journal of the Franklin Institute, 351(4):1866-1901, 2014.

[12] E. Bernuau, A. Polyakov, D. Efimov, and W. Perruquetti. Verification of iss, iiss and ioss properties applying weighted homogeneity. Systems \& Control Letters, 62(12):1159-1167, 2013.

[13] S.P. Bhat and D.S. Bernstein. Geometric homogeneity with applications to finite-time stability. Mathematics of Control, Signals and Systems, 17:101-127, 2005.

[14] I.I. Blekhman. Vibrational Mechanics. Fizmatlit, Moscow, 1994. in Russian.

[15] V.S. Bokharaie, O. Mason, and M. Verwoerd. D-stability and delay-independent stability of homogeneous cooperative systems. IEEE Trans. Automatic Control, 55(12):2882-2885, 2010 .

[16] J. Diblik. Asymptotic equilibrium for homogeneous delay linear differential equations with l-perturbation term. Nonlinear Analysis, Theory, Methods $\&$ Applicarions, 30(6):39273933, 1997.

[17] D. Efimov and A. Aleksandrov. Analysis of robustness of homogeneous systems with time delays using Lyapunov-Krasovskii functionals. Int. J. Robust and Nonlinear Control, 2020.

[18] D. Efimov, L. Panteley, and A. Loria. On input-to-output stability of switched nonlinear systems. In Proc. 17th IFACWorld Congress, pages 3647-3652, 2008.

[19] D. Efimov and W. Perruquetti. Oscillations conditions in homogenous systems. In Proc. NOLCOS'10, pages 1379-1384, Bologna, 2010.

[20] D. Efimov and W. Perruquetti. Conditions of oscillations and multi-homogeneity. Mathematics of Control, Signals, and Systems, 28(3):1-37, 2016.

[21] D. Efimov, W. Perruquetti, and M. Petreczky. On necessary conditions of instability and design of destabilizing controls. In Proc. 53rd IEEE CDC, LA, 2014.

[22] D. Efimov, W. Perruquetti, and J.-P. Richard. Development of homogeneity concept for time-delay systems. SIAM J. Control Optim., 52(3):1403-1808, 2014.

[23] D. Efimov, A. Polyakov, W. Perruquetti, and J.-P. Richard. Weighted homogeneity for time-delay systems: Finite-time and independent of delay stability. IEEE Trans. Automatic Control, 61(1):210-215, 2016.

[24] D. Efimov, R. Ushirobira, J.A. Moreno, and W. Perruquetti. Homogeneous lyapunov functions: from converse design to numerical implementation. SIAM J. Control Optimization, 56(5):3454-3477, 2018. 
[25] A.F. Filippov. Stability of differential equations with discontinuous and multi-valued righthand parts. Differential equations, 15(6):1018-1027, 1979.

[26] E. Fridman. Introduction to Time-Delay Systems: Analysis and Control. Birkhäuser, Basel, 2014.

[27] O.V. Gendelman and C.H. Lamarque. Dynamics of linear oscillator coupled to strongly nonlinear attachment with multiple states of equilibrium. Chaos, Solitons 85 Fractals, 24(2):501-509, 2005.

[28] K. Gu, V.L. Kharitonov, and J. Chen. Stability of Time-Delay Systems. Control Engineering. Birkhäuser, Boston, 2003.

[29] J.K. Hale. Theory of Functional Differential Equations. Springer-Verlag, 1977.

[30] J.P. Hespanha and A.S. Morse. Stability of switched systems with average dwell-time. In Proc. 38th IEEE Conferenceon Decision and Control, pages 2655-2660, 1999.

[31] Laurentiu Hetel, Christophe Fiter, Hassan Omran, Alexandre Seuret, Emilia Fridman, Jean-Pierre Richard, and Silviu Iulian Niculescu. Recent developments on the stability of systems with aperiodic sampling: An overview. Automatica, 76:309-335, 2017.

[32] Z.P. Jiang, Y. Lin, and Y. Wang. Stability analysis for switched systems with ISS and unstable time-delayed subsystems. In Proc. Chinese Control and Decision Conference, pages 905-909, 2016.

[33] A.Ya. Kanevskiy and L.Ye. Reyzign. Design of homogeneous Lyapunov-Krasovskii functions. Differential equations, 9(2):251-259, 1973.

[34] I. Karafyllis and M. Krstic. Predictor Feedback for Delay Systems: Implementations and Approximations, chapter Discrete-time systems, pages 251-278. Springer, 2017.

[35] I. Karafyllis, M. Malisoff, F. Mazenc, and P. Pepe, editors. Recent Results on Nonlinear Delay Control Systems, volume 4 of Advances in Delays and Dynamics. Springer International Publishing, 2016.

[36] M. Kawski. Homogeneous feedback stabilization, volume 7 of Progress in systems and control theory: New trends in systems theory. Birkhäuser, 1991.

[37] V.B. Kolmanovsky and V.R. Nosov. Stability of functional differential equations. CA: Academic, San Diego, 1986.

[38] I Kovacic. Forced vibrations of oscillators with a purely nonlinear powerform restoring force. J. Sound Vib., 330:4313-4327, 2011.

[39] A. Levant. Homogeneity approach to high-order sliding mode design. Automatica, 41(5):823-830, 2005.

[40] D. Liberzon. Switching in Systems and Control. Systems \& Control: Foundations \& Applications. Birkhäuser, 2003.

[41] D. Liberzon and A.S. Morse. Basic problems in stability and design of switched systems. IEEE Control Systems Magazine, 19(5):59-70, 1999. 
[42] Guopin Liu, Changchun Hua, and Xinping Guan. Asynchronous stabilization of switched neutral systems: A cooperative stabilizing approach. Nonlinear Analysis: Hybrid Systems, 33:380-392, 2019.

[43] A Luongo and D Zulli. Nonlinear energy sink to control elastic strings: The internal resonance case. Nonlinear Dyn., 81(1-2):425-435, 2015.

[44] F. Mazenc, M. Malisoff, and H. Özbay. Stability and robustness analysis for switched systems with time-varying delays. SIAM Journal on Control and Optimization, 56(1):158$182,2018$.

[45] F. Mazenc, S. Mondie, and S.-I. Niculescu. Global asymptotic stabilization for chains of integrators with a delay in the input. In Proc. 40th IEEE Conference on Decision and Control, volume 2, pages 1843-1848, 2001.

[46] A. Polyakov, D. Efimov, E. Fridman, and W. Perruquetti. On homogeneous distributed parameter systems. IEEE Trans. Automatic Control, 61(11):3657-3662, 2016.

[47] J.-P. Richard. Time-delay systems: an overview of some recent advances and open problems. Automatica, 39:1667-1694, 2003.

[48] L. Rosier. Homogeneous Lyapunov function for homogeneous continuous vector field. SystemsÉControl Lett., 19:467-473, 1992.

[49] T. Sanchez, D. Efimov, A. Polyakov, J.A. Moreno, and W. Perruquetti. On homogeneity of discrete-time systems: stability and convergence rates. Int. J. Robust and Nonlinear Control, 29(8):2406-2421, 2019.

[50] J. Schiffer, E. Fridman, R. Ortega, and J. Raisch. Stability of a class of delayed portHamiltonian systems with application to microgrids with distributed rotational and electronic generation. Automatica, 74:71-79, 2016.

[51] L. Vu, D. Chatterjee, and D. Liberzon. Input-to-state stability of switched systems and switching adaptive control. Automatica, 43:639-646, 2007.

[52] L. Vu and D. Liberzon. Supervisory control of uncertain linear time-varying systems. IEEE Transactions on Automatic Control, 56(1):27-42, 2011.

[53] L. Vu and K.A. Morgansen. Stability of time-delay feedback switched linear systems. IEEE Transactions on Automatic Control, 55(10):2385-2390, 2010.

[54] P. Yan and H. Öbay. Stability analysis of switched time-delay systems. SIAM Journal on Control and Optimization, 47(2):936-949, 2008.

[55] A. Zappavigna, P. Colaneri, J. Geromel, and R. Shorten. Dwell time analysis for continuous-time switched linear positive systems. In Proc. American Control Conference, Baltimore, MD, USA, 2010.

[56] J. Zhang and D. Efimov. A Lyapunov-Razumikhin condition of ISS for switched time-delay systems underaverage dwell time commutation. In Proc. 21st IFAC World Congress, 2020.

[57] Y. Zhang, X. Liu, and X. Shen. Stability of switched systems with time delay. Nonlinear Analysis: Hybrid Systems, 1(1):44-58, 2007. 
[58] Dianhao Zheng, Hongbin Zhang, J. Andrew Zhang, Weixing Zheng, and Steven W. Su. Stability of asynchronous switched systems with sequence-based average dwell time approaches. Journal of the Franklin Institute, 357(4):2149-2166, 2020.

[59] K. Zimenko, D. Efimov, A. Polyakov, and W. Perruquetti. A note on delay robustness for homogeneous systems with negative degree. Automatica, 79(5):178-184, 2017.

[60] V.I. Zubov. On systems of ordinary differential equations with generalized homogenous right-hand sides. Izvestia vuzov. Mathematica., 1:80-88, 1958. in Russian. 